\title{
Finitude homotopique et isotopique des structures de contact tendues
}

\author{
Vincent Colin, Emmanuel Giroux et Ko Honda
}

Résumé. Soit $V$ une variété close de dimension 3. Dans cet article, on montre que les classes d'homotopie de champs de plans sur $V$ qui contiennent des structures de contact tendues sont en nombre fini et que, si $V$ est atoroïdale, les classes d'isotopie des structures de contact tendues sur $V$ sont elles aussi en nombre fini.

Mots clés : structure de contact tendue, champ de plans, homotopie, isotopie, conjugaison, triangulation, surface branchée.

Codes AMS : 57M50, 53C15.

Le but de cet article est de démontrer les deux théorèmes suivants ainsi que quelques uns de leurs avatars :

Théorème 0.1. Sur toute variété close de dimension 3, les classes d'homotopie de champs de plans qui contiennent des structures de contact tendues sont en nombre fini.

Théorème 0.2. Sur toute variété close et atoroïdale de dimension 3 , les classes d'isotopie des structures de contact tendues sont en nombre fini.

Ces résultats s'inscrivent dans un contexte qu'on rappelle brièvement. D'abord, les structures de contact tendues sont officiellement nées dans [El2] où Y. Eliashberg établit à leur sujet le théorème de finitude homologique suivant : sur toute variété close $V$ de dimension 3, seul un nombre fini de classes de cohomologie entières de degré 2 sont réalisables comme les classes d'Euler de structures de contact tendues (orientées). Dans ce même article, le théorème de finitude homotopique 0.1 apparaît aussi explicitement - Theorem 2.2.2 - mais l'argument proposé pour sa démonstration est incomplet. Dans [KM], par l'étude des monopoles de Seiberg-Witten, P. Kronheimer et T. Mrowka obtiennent l'analogue du théorème 0.1 pour les structures de contact remplissables (au sens le plus faible qui soit). D'après un théorème de M. Gromov [Gr] et Y. Eliashberg [El3], toute structure de contact remplissable est tendue mais bien des structures de contact tendues ne sont, en revanche, pas remplissables [EH2, LS], de sorte que le théorème 0.1 ne découle pas des résultats de $[\mathrm{KM}]$.

Un autre théorème de El2 prouve que la sphère $\mathbf{S}^{3}$ porte une seule structure de contact tendue à isotopie (et changement d'orientation) près. Ceci suggère que les classes d'isotopie des structures de contact tendues pourraient être en nombre fini sur toute variété close de dimension 3 [El2, conjecture 8.6.1] mais les résultats de [Gi3, Ka] réfutent cette hypothèse. Ils montrent en effet qu'une modification de Lutz répétée sur un tore incompressible peut produire une infinité de structures de contact tendues deux à deux non conjuguées, d'où la conjecture suivante de Gi4] : les structures de contact tendues sur une variété close de dimension 3 forment un nombre fini de classes d'isotopie si et 
seulement si la variété est atorö̈dale, i.e. ne contient aucun tore plongé incompressible. Cette conjecture est à présent avérée grâce au théorème 0.2 ci-dessus et au résultat suivant démontré indépendamment dans [Co3] et dans [HKM] (travail commun avec W. Kazez et G. Matić) :

Théorème 0.3. Sur toute variété de dimension 3 close, orientée, torö̈dale et irréductibl凹, les structures de contact tendues forment une infinité de classes de conjugaison.

Les méthodes développées dans le présent article permettent par ailleurs de préciser ce résultat en tenant compte de la torsion, invariant des structures de contact introduit dans Gi4 et défini comme suit :

Définition 0.4. On appelle torsion d'une structure de contact $\xi$ sur une variété close $V$ de dimension 3 la borne supérieure des entiers $n \in \mathbb{N}$ pour lesquels on peut plonger dans $(V, \xi)$ le produit $\mathbf{T}^{2} \times[0,2 n \pi]$ muni de la structure de contact d'équation $\cos \theta d x_{1}-\sin \theta d x_{2}=0$, où $(x, \theta) \in \mathbb{R}^{2} / \mathbb{Z}^{2} \times[0,2 n \pi]$.

En vertu du théorème de Y. Eliashberg qui les classifie, les structures de contact non tendues - aussi dites vrillées - ont une torsion infinie. En outre, sur une variété atoroïdale et irréductible, les structures de contact tendues ont une torsion nulle. Pour ce qui concerne les variétés toroïdales, les démonstrations du théorème ci-dessus établissent en fait que, lorsqu'elles sont irréductibles, elles admettent des structures de contact tendues de torsion finie arbitrairement grande. Cela dit, la réponse à la question suivante reste inconnue :

Question 0.5. Les structures de contact tendues ont-elles toutes une torsion finie?

Le théorème qu'on obtient ici est le suivant :

Théorème 0.6. Sur toute variété close de dimension 3, les structures de contact tendues et de torsion égale à un entier fixé ne forment qu'un nombre fini d'orbites sous l'action du groupe engendré par les difféomorphismes isotopes à l'identité et les twists de Dehn le long de tores.

Ce théorème entraîne le théorème 0.2 puisque, sur une variété $V$ atoroïdale, tout twist de Dehn le long d'un tore est isotope à l'identité. Sur une variété torö̈dale en revanche, les structures de contact tendues de torsion (finie) fixée peuvent très bien former une infinité de classes d'isotopie, comme le montre l'exemple des fibrés en cercles au-dessus des surfaces Gi5, Ho2].

La clé des théorèmes $0.1,0.2$ et 0.6 est le résultat suivant qui montre que les structures de contact tendues sur une variété close sont engendrées, à partir d'un nombre fini d'entre elles, par des modifications de Lutz (voir la partie 1.4 pour la définition de cette opération) :

Théorème 0.7. Sur toute variété close $V$ de dimension 3 , il existe un nombre fini de structures de contact $\xi_{1}, \ldots, \xi_{n}$ et, pour chaque entier $i \in\{1, \ldots, n\}$, un nombre fini de tores $T_{1}^{i}, \ldots, T_{k_{i}}^{i}$ transversaux $\grave{a} \xi_{i}$ tels que toute structure de contact tendue $\xi$ sur $V$, ̀̀ isotopie près, s'obtienne à partir d'une des structures $\xi_{i}$ par une modification de Lutz de coefficients $n_{j}^{i}(\xi) \in \mathbb{N}$ le long des tores $T_{j}^{i}, 1 \leq j \leq k_{i}$.

\footnotetext{
${ }^{1}$ Sans cette hypothèse, il se peut que la variété ne porte aucune structure de contact tendue (voir EH1.
} 
Un point crucial néanmoins - qui empêche de déduire formellement le théorème 0.6 du théorème 0.7 - est ici que les tores $T_{j}^{i}$ fournis par ce dernier ne sont a priori pas disjoints - même pour $i$ fixé. Du coup, l'ordre dans lequel on effectue les modifications de Lutz a une incidence.

Tous ces résultats fournissent une classification géométrique grossière des structures de contact tendues en dimension 3. Il serait intéressant de la préciser en donnant par exemple des estimations effectives pour les nombres dont on se borne ici à prouver qu'ils sont finis.

Les preuves qu'on donne dans ce texte s'étendent au cas des variétés compactes à bord, pour peu qu'on impose un germe de structure de contact le long du bord. Par exemple, le théorème 0.6 devient :

Théorème 0.8. Soit $V$ une variété compacte à bord de dimension 3 et $\zeta$ un germe de structure de contact le long de $\partial V$. Les structures de contact tendues égales à $\zeta$ près de $\partial V$ et de torsion égale à un entier fixé ne forment qu'un nombre fini d'orbites sous l'action du groupe engendré par les difféomorphismes isotopes à l'identité relativement au bord et les twists de Dehn le long de tores inclus dans $\operatorname{Int}(V)$.

Grâce au travail de Y. Eliashberg et W. Thurston [ET] qui procure, en dimension 3, une méthode générale pour déformer un feuilletage par surfaces en une structure de contact, le résultat suivant de D. Gabai [Ga] se déduit du théorème 0.1 :

Corollaire 0.9. Sur toute variété de dimension 3 close et orientable, les classes d'homotopie de champs de plans qui contiennent un feuilletage sans composantes de Reeb sont en nombre fini.

Avant D. Gabai, P. Kronheimer et T. Mrowka ont démontré ce théorème dans [KM] pour une classe plus restreinte de feuilletages - les feuilletages tendus - comme une conséquence de leur travail sur les structures de contact remplissables. En fait, un des résultats principaux de [ET] dit que tout feuilletage tendu sur une variété close orientable peut être déformé en une structure de contact remplissable. Par suite, la finitude homotopique des structures de contact remplissables implique celle des feuilletages tendus. Le corollaire 0.9 découle pareillement du théorème 0.1 et d'un résultat de [Co4 qui montre que, toujours sur une variété close et orientable, tout feuilletage sans composantes de Reeb se laisse déformer en une structure de contact tendue.

Dans Ga], D. Gabai démontre le corollaire 0.9 par une méthode géométrique fondée sur les idées classiques de H. Kneser [Kn], W. Haken [Ha] et F. Waldhausen [Wa] mais utilisant de plus un processus infini de mise sous forme normale dû̀ à $\mathrm{M}$. Brittenham $[\mathrm{Br}]$. Cette méthode lui permet d'atteindre aussi un analogue du théorème 0.2 qui, pour le coup, semble loin d'en être un corollaire.

Enfin, la version relative 0.8 du théorème 0.6 donne une classification grossière des nœuds legendriens dans la sphère $\mathbf{S}^{3}$ munie de sa structure de contact standard :

Théorème 0.10. Dans la sphère de contact standard de dimension 3, les nœuds legendriens ayant une classe d'isotopie lisse et un invariant de Thurston-Bennequin imposés - arbitrairement - forment un nombre fini de classes d'isotopie legendrienne.

Pour démontrer les résultats énoncés ci-dessus, la méthode qu'on adopte consiste à mettre chaque structure de contact tendue sur $V$, par isotopie, en position « adaptée » et 
« minimale » par rapport à une triangulation fixe $\Delta$ de $V$ (sections 2.1 et 2.2.). Ensuite, grâce à la classification des structures de contact tendues sur la boule [El2] et à des manipulations de surfaces convexes Gi1, on observe que chaque structure de contact tendue obtenue est entièrement décrite par une donnée combinatoire : le découpage des faces de $\Delta$. Il s'agit, sur chaque 2 -simplexe $F$, d'une collection finie d'arcs simples, propres, disjoints et évitant les sommets, leur union n'étant bien définie qu'à une isotopie près de $F$ relative aux sommets. Ces arcs (à cause des propriétés de la triangulation et des structures de contact), s'organisent en familles d'arcs parallèles, elles-mêmes contenues dans un nombre fini de «domaines fibrés » (sections 2.3 et 2.4). En dehors de ces domaines, les structures sont sous contrôle (théorème 2.1). On établit alors une correspondance entre les structures de contact tendues « ajustées » à un même domaine fibré $M$ et les surfaces « portées » par $M$. C'est là qu'apparaissent les tores générateurs du théorème 0.7 (section 3). Pour démontrer la finitude isotopique, il faut encore simplifier le domaine $M$ pour le faire ressembler à un fibré en cercles au-dessus d'une surface (section 4). On applique alors un théorème classifiant les structures tendues portées par un fibré en cercles [Gi4, Gi5, Ho2] pour conclure. Enfin, on donne la preuve du théorème 0.10 dans la section 5. La section 1 est consacrée à la présentation des outils nécéssaires à la réalisation de ce programme.

Remerciements. Le travail présenté ici est, pour l'essentiel, le fruit de discussions entamées à l'American Institute of Mathematics - Palo Alto, USA - à l'automne 2000 et poursuivies à l'Institut des Hautes Études Scientifiques - Bures-sur-Yvette, France - au printemps 2001. Les auteurs remercient vivement ces deux organismes pour leur accueil et leur aide matérielle. Ils remercient également le CNRS, la NSF, la Fondation Alfred Sloan et l'Institut Universitaire de France pour avoir en partie financé leurs recherches. Ils tiennent enfin à adresser un grand merci à François Laudenbach pour sa relecture attentive d'une ébauche du texte.

\section{Outils de géométrie de contact et de topologie}

On présente ici succintement les outils de géométrie de contact et de topologie qui serviront pour démontrer les théorèmes annoncés dans l'introduction.

\subsection{Structures de contact tendues / vrillées}

Dans ce texte, $V$ désigne toujours une variété compacte et orientée de dimension 3 et chaque structure de contact $\xi$ qu'on considère sur $V$ est directe et (co-) orientée. En d'autres termes, il existe sur $V$ une 1-forme $\alpha$ - unique à multiplication près par une fonction positive - dont $\xi$ est le noyau coorienté et dont le produit avec $d \alpha$ est partout un élément de volume positif relativement à l'orientation de $V$.

Sur une variété close, les structures de contact forment au plus une infinité dénombrable de classes d'isotopie. Cela découle du théorème de Gray qui montre qu'elles sont stables dans la topologie $\mathcal{C}^{1}:$ si $\xi_{s}, s \in[0,1]$, est un chemin de structures de contact sur une variété close $V$, il existe une isotopie $\phi_{s}$ de $V$ partant de l'identité $\left(\phi_{0}=\mathrm{id}\right)$ et vérifiant $\phi_{s *} \xi_{0}=\xi_{s}$ pour tout $s$ - en dimension 3, on dispose même d'un résultat beaucoup plus fort de stabilité $\mathcal{C}^{0}$ Co2]. Un autre fait important est qu'une variété de contact close $(V, \xi)$ possède un grand nombre d'isotopies de contact. Plus explicitement, la projection $T V \rightarrow T V / \xi$ met en bijection les champs de vecteurs sur $V$ qui préservent $\xi$ 
et les sections du fibré quotient $T V / \xi \rightarrow V$. Autrement dit, si $\xi$ est le noyau d'une forme de contact $\alpha$, toute fonction lisse $u: V \rightarrow \mathbb{R}$ détermine un unique champ de vecteurs $\nabla_{\alpha} u$ qui conserve $\xi$ et sur lequel $\alpha$ vaut $u$. Le problème du prolongement des isotopies - ou des champs de vecteurs - de contact se ramène en particulier à un problème de prolongement de fonctions.

Un disque vrillé $D$ dans une variété de contact $(V, \xi)$ est un disque plongé dans $V$ et dont le plan tangent $T_{p} D$, en chaque point $p$ du bord $\partial D$, coïncide avec le plan de contact $\xi_{p}$. La structure de contact $\xi$ est dite vrillée ou tendue selon qu'un disque vrillé existe ou non à l'intérieur de $V$. Cette dichotomie a deux raisons d'être majeures. En premier lieu, un théorème de Y. Eliashberg [El1] établit la classification isotopique des structures de contact vrillées sur toute variété close : chaque classe d'homotopie de champs de plans tangents contient une et une seule classe d'isotopie de structures de contact vrillées. D'autre part, les disques vrillés s'interprètent comme des cycles évanescents. Plus précisément, Y. Eliashberg et W. Thurston ont proposé dans [ET] la définition suivante : un cycle évanescent pour un champ de plans quelconque $\zeta$ est un disque plongé $D$ qui est partout tangent à $\zeta$ le long de son bord mais n'est pas isotope à un disque intégral de $\zeta$ relativement à $\partial D$. Avec ce langage, une structure de contact (resp. un feuilletage) sans cycles évanescents est une structure de contact tendue (resp. un feuilletage sans composantes de Reeb : théorème de Novikov).

La classification des structures de contact tendues n'est connue que sur peu de variétés, essentiellement celles que des découpages successifs le long de tores et d'anneaux ramènent à des boules. Sur la boule, un théorème de Y. Eliashberg [El2] affirme qu'il n'y a essentiellement qu'une structure tendue.

Théorème 1.1. Des structures de contact tendues sur la boule qui impriment sur le bord le même champ de droites singulier (ou, autrement dit, le même feuilletage caractéristique : voir plus loin) sont isotopes relativement au bord.

Dans la suite, une variété close et orientée $V$ de dimension 3 étant donnée, $\mathcal{S C T}(V)$ désigne l'espace de ses structures de contact tendues et on dit qu'un sous-ensemble $\mathcal{X}$ de $\mathcal{S C T}(V)$ est complet s'il représente toutes les classes d'isotopie, c'est-à-dire s'il possède (au moins) un point dans chaque composante connexe de $\mathcal{S C T}(V)$.

\subsection{Surfaces convexes}

La théorie des surfaces convexes [Gi1] fournit, pour les variétés de contact, des techniques de découpage et de collage le long de surfaces. On peut brièvement la résumer ainsi : au voisinage d'une surface générique, la géométrie d'une structure de contact est entièrement inscrite dans une multi-courbe tracée sur la surface.

Toute surface $S$ plongée dans une variété de contact $(V, \xi)$ hérite d'un feuilletage caractéristique noté $\xi S$ et engendré par le champ singulier de droites $\xi \cap T S$. Ce feuilletage détermine totalement le germe de $\xi$ le long de $S$, à isotopie de $V$ préservant $S$ et $\xi S$ près. Lorsque $\xi$ et $S$ sont orientées, $\xi S$ l'est aussi. Concrètement, si $\xi$ est le noyau de $\alpha$ et si $\omega$ est une forme d'aire sur $S$, le feuilletage $\xi S$ est constitué des courbes intégrales orientées du champ de vecteurs $\eta$ dont le produit intérieur avec $\omega$ est égal à la 1-forme induite par $\alpha$ sur $S$. Une singularité de $\xi S$ est un point de tangence entre $\xi$ et $S$ et a donc un signe : elle est positive ou négative selon que les orientations de $\xi$ et $S$ coïncident ou non. Ce signe est également celui de la divergence de $\eta$. Les singularités de $\xi S$ sont aussi 
appelées points complexes de $S$. Un point complexe est dit elliptique (resp. hyperbolique) si c'est une singularité isolée de $\xi S$ qui est de type foyer (resp. selle), c'est-à-dire d'indice 1 (resp. -1 ).

Soit $S$ une surface compacte, orientée, plongée dans $(V, \xi)$ et à bord vide ou legendrien - une courbe legendrienne est une courbe partout tangente à $\xi$. On dit que $S$ est $\xi$-convexe si elle a un épaississement

$$
U=S \times \mathbb{R} \supset S=S \times\{0\}
$$

dans lequel l'action de $\mathbb{R}$ par translations (action engendrée par le champ de vecteurs $\partial_{t}$, $t \in \mathbb{R}$ ) préserve $\xi$. Un tel épaississement $U$ est dit homogène.

Une surface $\xi$-convexe possède non seulement un épaississement homogène mais tout un «système fondamental» de tels voisinages. En d'autres termes, tout voisinage de $S$ contient un voisinage homogène de $S$. En effet, si $\nu$ est la section de $T V / \xi$ associée au champ de vecteurs $\partial_{t}$ dans un épaississement homogène $U=S \times \mathbb{R}$, le fait que $\partial S$ soit legendrien assure que le champ de vecteurs de contact associé à toute section du type $f \nu$, où $f$ est une fonction qui ne dépend que de $t$, reste tangent à $\partial U=\partial S \times \mathbb{R}$. En effet, pour $\xi=\operatorname{ker} \alpha$ et $v=\alpha(\nu)$, si on cherche le champ de contact $\nabla_{\alpha}(f v)$ (défini dans la section 1.1) sous la forme $\nabla_{\alpha}(f v)=f \frac{\partial}{\partial t}+X_{0}$, avec $X_{0} \in \xi$, cela résulte de la formule

$$
\left.i_{X_{0}} d \alpha\right|_{\xi}=-\left.v d f\right|_{\xi}
$$

La $\xi$-convexité est ainsi une propriété locale qui, pour peu que $S$ ne touche pas $\partial V$, se lit directement sur le feuilletage caractéristique $\xi S$ comme expliqué ci-dessous.

On considère sur $S$ un feuilletage singulier $\sigma$-famille des courbes intégrales orientées d'un champ de vecteurs - et une multi-courbe $\Gamma$ - union finie de courbes fermées, simples et disjointes. On dit que $\Gamma$ est une courbe de découpage de $\sigma$ (où découpe $\sigma$ ) si $\sigma$ est transversal à $\Gamma$ et est porté par un champ de vecteurs qui, sur chaque composante de $S \backslash \Gamma$, ou bien dilate l'aire et sort le long de $\Gamma$, ou bien contracte l'aire et rentre le long de $\Gamma$. Une telle courbe, si elle existe et si $\sigma$ est tangent à $\partial S$, est unique à isotopie près parmi les courbes de découpage.

Le lemme qui suit est établi dans Gi1 pour les surfaces closes mais sa démonstration s'étend au cas des surfaces à bord legendrien :

Lemme 1.2. Soit $\xi$ une structure de contact sur $V$ et $S \subset \operatorname{Int}(V)$ une surface compacte, orientée et à bord legendrien. Pour que $S$ soit $\xi$-convexe, il faut et il suffit que son feuilletage caractéristique $\xi S$ soit découpé par une multi-courbe. De plus, cette condition est remplie dès que les propriétés suivantes sont satisfaites:

1. chaque demi-orbite de $\xi S$ a pour ensemble limite une singularité ou une orbite fermée ;

2. les orbites fermées de $\xi S$ sont toutes hyperboliques;

3. aucune orbite (orientée) de $\xi S$ ne va d'une singularité négative à une singularité positive.

Remarque 1.3. La dernière des trois propriétés ci-dessus mérite un commentaire : une orbite de $\xi S$ qui va d'une singularité négative à une singularité positive est un arc legendrien sur $S$ le long duquel $\xi$ effectue globalement autour de $S$ un demi-tour dans le sens contraire des aiguilles d'une montre. 
Soit $S$ une surface compacte orientée plongée dans $(V, \xi)$. Compte tenu du théorème de M. Peixoto qui affirme qu'un feuilletage singulier de $S$ est génériquement de type MorseSmale (et donc possède les propriétés 1, 2 et 3), le lemme 1.2 ci-dessus montre Gi1] que, si $S$ est close, on peut la rendre $\xi$-convexe en la perturbant par une isotopie arbitrairement $\mathcal{C}^{\infty}$-petite. De même, si $S$ est à bord legendrien et si aucune orbite de $\xi S$ sur $\partial S$ ne va d'une singularité négative vers une singularité positive (i.e. si, entre deux singularités consécutives de signes contraires sur $\partial S$, le demi-tour que $\xi$ fait autour de $S$ s'effectue globalement dans le sens des aiguilles d'une montre), on peut aussi la rendre $\xi$-convexe en la déplaçant par une isotopie relative au bord et arbitrairement $\mathcal{C}^{\infty}$-petite.

Soit maintenant $L$ une courbe legendrienne tracée sur $S$. On appelle nombre de Thurston-Bennequin de $L$ relatif à $S$ le nombre d'enroulement $\operatorname{tb}(L, S) \in \frac{1}{2} \mathbb{Z}$ de $\xi$ autour de $T S$ le long de $L$ (si $L$ est fermée, $\operatorname{tb}(L, S) \in \mathbb{Z}$ ), qui décompte algébriquement les points complexes de $S$ le long de $L$. On vérifie facilement que, quand $S$ est $\xi$-convexe et que les points extrêmes de $L$ sont complexes - si $L$ n'est pas fermée -,

$$
\operatorname{tb}(L, S)=-\frac{1}{2} \operatorname{Card}(L \cap \Gamma)
$$

où $\Gamma$ est une multi-courbe qui découpe $\xi S$. En particulier, chaque composante de $\partial S$ a un invariant de Thurston-Bennequin négatif ou nul. Inversement, si $S$ est une surface compacte à bord legendrien et si chaque composante de $\partial S$ a un nombre de ThurstonBennequin négatif ou nul, une isotopie arbitrairement $\mathcal{C}^{0}$-petite, relative au bord et à support dans un voisinage aussi petit qu'on veut de $\partial S$, permet de perturber $S$ en une surface dont aucune orbite du feuilletage caractéristique sur le bord ne lie une singularité négative à une singularité positive. Une isotopie arbitrairement $\mathcal{C}^{\infty}$-petite suffit ensuite pour obtenir une surface $\xi$-convexe. La condition portant sur le signe des nombres de Thurston-Bennequin des composantes de $\partial S$ est en outre aisée à satisfaire au moyen d'une isotopie, dite de stabilisation: pour toute courbe legendrienne $L$ tracée sur $S$, pour tout point $p \in L$, pour tout voisinage $N(p)$ de $p$ dans $V$ et pour tout $n \in \mathbb{N}$, il existe une isotopie $\left(\phi_{t}\right)_{t \in[0,1]}$ de $V$ à support dans $N(p)$ telle que $\phi_{1}(L)$ soit une courbe legendrienne et $\operatorname{tb}\left(\phi_{1}(L), \phi_{1}(S)\right)=\operatorname{tb}(L, S)-n$.

On suppose désormais que $S$ est $\xi$-convexe. Le choix d'un épaississement homogène $U=S \times \mathbb{R}$ détermine sur $S$ une multi-courbe $\Gamma_{U}$ qui découpe $\xi S$ : elle est formée des points de $S$ où le vecteur $\partial_{t}, t \in \mathbb{R}$, appartient au plan $\xi$. De plus, toute multi-courbe qui découpe $\xi S$ est le découpage associé à un certain épaisssissement homogène. Le lemme ci-dessous Gi1 montre que la multi-courbe $\Gamma_{U}$ concentre en fait toute la géométrie de contact de $U$ :

Lemme 1.4. [Lemme de réalisation de feuilletages] Soit $S$ une surface $\xi$-convexe, $U$ un épaississement homogène de $S$ et $\Gamma$ le découpage de $\xi S$ associé. Les feuilletages singuliers de $S$ tangents à $\partial S$ et découpés par $\Gamma$ constituent un espace contractile. De plus, pour tout feuilletage $\sigma$ dans cet espace, il existe un plongement $\phi: S \rightarrow U$ ayant les propriétés suivantes :

1. $\phi$ coïncide avec l'inclusion sur $\partial S$;

2. $\phi(S) \subset U=S \times \mathbb{R}$ est une surface transversale au champ de vecteurs $\partial_{t}, t \in \mathbb{R}$;

3. $\phi_{*} \sigma$ est le feuilletage caractéristique de $\phi(S)$.

On note que, vu la propriété $2, U$ est un épaississement homogène de $\phi(S)$ et que le découpage associé est l'intersection de $\phi(S)$ avec le cylindre $\Gamma \times \mathbb{R}$. En outre, $\phi$ est isotope à l'inclusion parmi les plongements $S \rightarrow U$ satisfaisant les conditions 1) et 2). 
Un corollaire direct du lemme ci-dessus est le résultat suivant [Gi1, exemple II.3.7] :

Lemme 1.5. [Lemme de réalisation legendrienne] Soit $S$ une surface $\xi$-convexe, $U$ un de ses épaississements homogènes, $\Gamma$ la multi-courbe de découpage de $\xi S$ associé et $C$ une multi-courbe sur $S$ transversale à $\Gamma$ et telle que chaque composante de $S \backslash C$ rencontre $\Gamma$. Il existe un plongement $\phi: S \rightarrow U$ ayant les propriétés suivantes :

1. $\phi$ cö̈ncide avec l'inclusion sur $\partial S$;

2. $\phi(S) \subset U=S \times \mathbb{R}$ est une surface transversale au champ de vecteurs $\partial_{t}, t \in \mathbb{R}$;

3. $\phi(C)$ est legendrienne et son nombre de Thurston-Bennequin $\operatorname{tb}(\phi(C), \phi(S))$ vaut $-\frac{1}{2} \operatorname{Card}(C \cap \Gamma)$.

\subsection{Rocades legendriennes}

Dans l'exploration d'une variété de contact $(V, \xi)$, les rocades (bypasses) [Ho1 servent à pousser l'investigation géométrique au-delà des domaines homogènes et à analyser les changements (chirurgies) élémentaires que subit le découpage d'une surface $\xi$-convexe quand on déforme celle-ci par une grande isotopie. Elles jouent ainsi, en topologie de contact, un rôle comparable à celui des disques de Whitney en topologie différentielle.

Une rocade (legendrienne) est un demi-disque $H$ plongé dans $(V, \xi)$ et ayant les propriétés suivantes :

- le bord de $H$ est legendrien et $H$ est $\xi$-convexe - en particulier, les plans $T_{p} H$ et $\xi_{p}$ coïncident en chaque coin $p$ de $H$;

- l'enroulement de $\xi$ autour de $T H$ vaut -1 le long d'un des deux $\operatorname{arcs}$ de $\partial H$, arc qu'on note $\partial_{-} H$, et 0 le long de l'autre, qu'on note $\partial_{\cap} H$ - l'invariant de ThurstonBennequin de $\partial H$ est donc égal à -1 et le découpage de $\xi H$ se réduit à un arc dont les deux extrémités se trouvent sur $\partial_{-} H$.

Une rocade matérialise ainsi une isotopie entre deux arcs legendriens reliant les mêmes points, $\partial_{-} H$ et $\partial_{\cap} H$, le second arc étant « plus court » que le premier au sens où la valeur absolue de l'enroulement de $\xi$ y est moindre.

Le lemme ci-dessous est un cas particulier du lemme de réalisation legendrienne 1.5. Il permet de dénicher une rocade à chaque fois qu'une surface $\xi$-convexe $S$ présente, dans son découpage $\Gamma$, un arc propre parallèle au bord (on dit aussi $\partial$-parallèle), c'est-à-dire bordant avec un arc de $\partial S$ un demi-disque $H_{0}$ dont l'intérieur est disjoint de $\Gamma$.

Lemme 1.6. Soit $S$ une surface $\xi$-convexe à bord legendrien lisse, $U$ un épaississement homogène de $S$ et $\Gamma$ le découpage de $\xi S$ associé. On suppose d'une part que, si $S$ est un disque, $\Gamma$ n'est pas connexe et d'autre part que l'adhérence d'une des composantes connexes de $S \backslash \Gamma$ est un demi-disque $D$ dont un des arcs du bord est dans $\partial S$ et l'autre dans $\Gamma$. On note $H$ un demi-disque voisinage de $D$ dans $S$ dont un arc du bord est dans $\partial S$ et dont l'intersection avec $\Gamma$ se réduit à l'arc $D \cap \Gamma$. Il existe alors un plongement $\phi: S \rightarrow U$ ayant les propriétés suivantes:

1. $\phi$ coïncide avec l'inclusion sur $\partial S$;

2. $\phi(S) \subset U=S \times \mathbb{R}$ est une surface transversale au champ de vecteurs $\partial_{t}, t \in \mathbb{R}$;

3. $\phi(\Gamma)$ est l'intersection de $\phi(S)$ avec le cylindre $\Gamma \times \mathbb{R} \subset U$;

4. $\phi(H)$ est une rocade legendrienne. 
On décrit maintenant l'attachement d'une rocade sur une surface $\xi$-convexe et son effet sur le découpage de celle-ci [Ho1].

Soit $S \subset V$ une surface $\xi$-convexe et $H$ une rocade attachée à $S$, c'est-à-dire vérifiant les propriétés suivantes :

- $H$ s'appuie transversalement sur $S$ le long de $\partial_{-} H$ et ne touche $S$ nulle part ailleurs ;

- il existe un découpage $\Gamma$ de $S$ qui passe par les extrémités de $\partial_{-} H$ (et automatiquement par le milieu de $\left.\partial_{-} H\right)$.

On oriente $S$ de façon que $H$ soit du côté positif et on note $N_{\varepsilon}$ le $\varepsilon$-voisinage de $H$ privé de ce qui déborde du côté négatif de $S$. Alors, pour $\varepsilon>0$ assez petit, il existe sur $V$ un champ de vecteurs de contact qui est transversal à la surface (lisse par morceaux)

$$
\operatorname{Adh}\left(\left(S \cup \partial N_{\varepsilon}\right) \backslash\left(S \cap N_{\varepsilon}\right)\right)
$$

et rentrant dans le quadrant extérieur à $N_{\epsilon}$ [Ho1]. On peut donc lisser cette surface transversalement audit champ de vecteurs pour obtenir une surface $\xi$-convexe $S^{\prime}$. Il existe clairement une isotopie de $S$ à $S^{\prime}$ et, si on l'utilise pour identifier l'une à l'autre ces surfaces, on peut décrire comme suit (à isotopie près) le découpage $\Gamma^{\prime}$ de $S^{\prime}$ en fonction du découpage $\Gamma$ de $S$ et de la base $\partial_{-} H$ de $H$. On prend sur $S$ un carré $R=[-1,1] \times[-1,1]$ (les coordonnées étant compatibles avec l'orientation) qui contient $\partial_{-} H=[-1,1] \times\{0\}$ et rencontre $\Gamma$ selon les trois segments $\{-1,0,1\} \times[-1,1]$. La multi-courbe $\Gamma^{\prime}$ s'obtient à partir de $\Gamma$ en retirant les segments

$$
\{-1\} \times[0,1], \quad\{0\} \times[-1,1] \text { et }\{1\} \times[-1,0]
$$

et en les remplaçant par

$$
[-1,0] \times\{1\}, \quad[-1,1] \times\{0\} \quad \text { et } \quad[0,1] \times\{-1\} .
$$

On nomme cette opération chirurgie de $\Gamma$ le long de $\partial_{-} H$. Dit autrement, si on regarde plutôt le complémentaire du découpage comme un coloriage de la surface en deux couleurs, le passage de $S$ à $S^{\prime}$ consiste à faire tourner, dans le carré $R$, le coloriage d'un angle $\pi / 2$ sans le changer en dehors.

\subsection{Modification de Lutz}

La modification de Lutz, définie ci-après, est une opération qui consiste à changer une structure de contact au voisinage d'un tore transversal.

Soit $\xi$ une structure de contact sur $V$ et $T$ un tore plongé dans $V$ transversalement à $\xi$. On note

$$
W=T \times[-1,1] \supset T=T \times\{0\}
$$

un voisinage tubulaire compact de $T$ dont les fibres sont des arcs legendriens dans $(V, \xi)$ et on paramètre $T$ par $\mathbb{R}^{2} / \mathbb{Z}^{2}$. Ainsi, la structure de contact $\xi$ admet dans $W$ une équation du type

$$
\cos \theta(x, t) d x_{1}-\sin \theta(x, t) d x_{2}=0, \quad(x, t) \in \mathbb{R}^{2} / \mathbb{Z}^{2} \times[-1,1],
$$

où la fonction $\theta: \mathbb{R}^{2} / \mathbb{Z}^{2} \times[-1,1] \rightarrow \mathbf{S}^{1}$ vérifie $\partial_{t} \theta>0$ en tout point.

Pour tout entier $n \geq 0$, soit $\rho_{n}:[-1,1] \rightarrow \mathbf{S}^{1}=\mathbb{R} / 2 \pi \mathbb{Z}$ la projection d'une fonction (faiblement) croissante $[-1,1] \rightarrow \mathbb{R}$ qui vaut 0 près de -1 et $n \pi$ près de 1 . La structure de contact définie sur $W$ par l'équation

$$
\cos \left(\theta(x, t)+\rho_{n}(t)\right) d x_{1}-\sin \left(\theta(x, t)+\rho_{n}(t)\right) d x_{2}=0, \quad(x, t) \in \mathbb{R}^{2} / \mathbb{Z}^{2} \times[-1,1],
$$


coïncide avec $\xi$ près de $T \times\{-1\}$ et avec $(-1)^{n} \xi$ près de $T \times\{1\}$. On peut donc la prolonger par $\xi$ en dehors de $W$ pour obtenir une structure de contact $\xi_{n}$ sur $V$ mais celle-ci n'est orientable que si $n$ est pair ou si $T$ sépare $V$. Par convention, on prend alors sur $\xi_{n}$ l'orientation qui épouse celle de $\xi$ près de $T \times\{1\}$. On dit que $\xi_{n}$ est obtenue à partir de $\xi$ par une modification de Lutz de coefficient $\frac{n}{2}$ le long de $T$.

Des calculs simples montrent que la classe d'isotopie de $\xi_{n}$ ne dépend que de $n$ (et pas du choix de $W$ ou de $\rho_{n}$ ) tandis que sa classe d'homotopie dans l'espace des champs de plans tangents est seulement fonction de la parité de $n$ et est notamment égale à celle de $\xi$ quand $n$ est pair.

Les deux exemples suivants permettent d'éclairer le rôle joué par les modifications de Lutz.

- Une modification de Lutz le long d'un tore compressible dont le feuilletage caractéristique est une suspension (ou plus généralement pour lequel le champ de droites tracé par $\xi$ sur $T$ est homotope à un champ constant dans la trivialisation habituelle de $T \cong \mathbb{R}^{2} / \mathbb{Z}^{2}$ ) donne toujours une structure de contact vrillée.

- Soit $T$ un tore dont le feuilletage caractéristique $\xi T$ est la réunion de deux composantes de Reeb qui pointent dans la même direction (en particulier le champ de ses tangentes n'est pas homotope à un champ de droites constant). Une modification de Lutz de coefficient 1 le long de $T$ donne une structure de contact conjuguée à la structure initiale $\xi$ par un twist de Dehn, et donc isotope à $\xi$ quand le tore est compressible. Pour s'en convaincre, il suffit de constater que faire pivoter les tangentes à $\xi T$ d'un angle $\theta$ conduit à un champ de droites dont le feuilletage intégral se déduit de $\xi T$ par une isotopie qui déplace les orbites périodiques. Lorsque $\theta$ varie entre 0 et $2 \pi$, les positions successives prises par une orbite périodique $\gamma$ de $\xi T$ forment un feuilletage de $T$.

\subsection{Surfaces branchées}

Soit $G_{0}, G_{1}, G_{2}$ les graphes respectifs dans $\mathbb{R}^{3}=\mathbb{R}^{2} \times \mathbb{R}$ des fonctions $f_{0}, f_{1}, f_{2}$ définies sur $\mathbb{R}^{2}$ par

$$
f_{0}(x, y)=0, \quad f_{1}(x, y)=\left\{\begin{array}{ll}
0 & \text { si } x \geq 0, \\
e^{1 / x} & \text { si } x<0,
\end{array} \quad \text { et } \quad f_{2}(x, y)=-f_{1}(y, x) .\right.
$$

On note $\mathbf{X}$ l'intersection de $G_{0} \cup G_{1} \cup G_{2}$ avec le demi-espace $\{x \geq-1\}$. Un homéomorphisme d'un ouvert $U$ de $\mathbf{X}$ sur un autre est dit lisse si sa restriction à chaque intersection $U \cap G_{i}$, $0 \leq i \leq 2$, est lisse comme application à valeurs dans $\mathbb{R}^{3}$.

Une surface branchée est un espace topologique $X$ séparé, de type dénombrable et localement modelé sur $\mathbf{X}$ par des cartes - c'est-à-dire dont chaque point possède un voisinage homéomorphe à un ouvert de $\mathbf{X}$ - avec des changements de cartes lisses. On nomme

- bord de $X$ l'ensemble $\partial X$ des points que les cartes appliquent dans l'intersection $\mathbf{X} \cap\{x=-1\}$ - cet ensemble est un graphe doté d'une tangente en tout point, autrement dit un réseau ferroviaire;

- point triple tout point de $X$ que les cartes envoient sur $(0,0,0)$;

- point double tout point de $X$ que les cartes appliquent dans $\mathbf{X} \cap\{x y=0\}$ sur un point autre que $(0,0,0)$;

- point régulier tout point de $X$ qui n'est ni double ni triple. 
Cette définition engendre une notion naturelle d'applications lisses d'une surface branchée dans une autre ou entre une surface branchée et une variété, d'où (par exemple) une notion de courbes immergées dans une surface branchée et de surfaces branchées plongées dans une variété.

Les sous-ensembles $X_{0}, X_{1}$ et $X_{2}$ constitués des points respectivement réguliers, doubles et triples forment une stratification de $X$. L'ensemble $X_{0}$, qu'on note aussi $\operatorname{Reg}(X)$, est appelé partie régulière de $X$ et son complémentaire $\Theta=X_{1} \cup X_{2}$ le lieu singulier. Ce lieu est une multi-courbe immergée à points doubles ordinaires (les points triples de $X$ ). L'ensemble $X_{1}$ est de plus naturellement coorienté : par convention, son côté positif est celui où $X$ a un seul feuillet. La direction donnée par cette coorientation est nommée direction de branchement. Enfin, les composantes connexes de l'ensemble $X_{0}=\operatorname{Reg}(X)$ sont appelées strates régulières ou secteurs de $\operatorname{Reg}(X)$.

\subsection{Domaines fibrés}

Les surfaces branchées apparaissent souvent, de façon naturelle, comme des quotients de variétés - à bord anguleux - par certains feuilletages de codimension 2.

A titre de premier exemple, on considère trois copies $P_{0}, P_{1}$ et $P_{2}$ du plan $\mathbb{R}^{2}$ et on met sur leur union la relation d'équivalence engendrée par les règles suivantes :

- un point $\left(x_{0}, y_{0}\right) \in P_{0}$ est équivalent à un point $\left(x_{1}, y_{1}\right) \in P_{1}$ si et seulement si $x_{0}=x_{1}, y_{0}=y_{1}$ et $x_{0} \leq 0$;

- un point $\left(x_{0}, y_{0}\right) \in P_{0}$ est équivalent à un point $\left(x_{2}, y_{2}\right) \in P_{2}$ si et seulement si $x_{0}=x_{2}, y_{0}=y_{2}$ et $y_{0} \leq 0$.

Le quotient de $P_{0} \cup P_{1} \cup P_{2}$ par cette relation est une surface branchée difféomorphe à l'union $G_{0} \cup G_{1} \cup G_{2}$ des trois graphes décrits au début de la partie précédente (voir la figure 1).
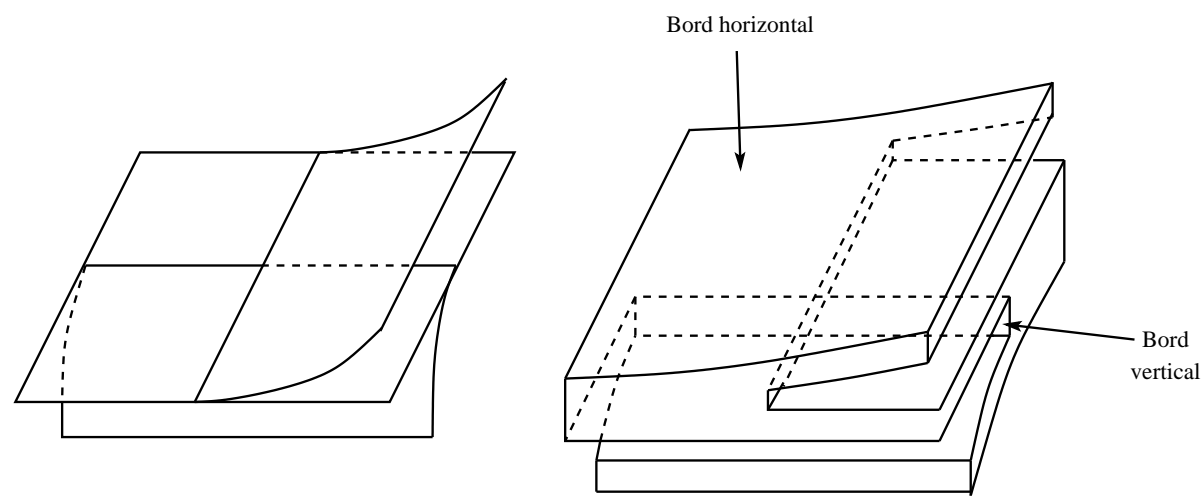

FIG. 1 - Surface branchée et domaine fibré.

Soit $M$ une variété compacte, orientable, à bord anguleux - en fait, ayant des arêtes lisses mais pas de coins - et munie d'un feuilletage $\tau$ en intervalles compacts. On dit que $M$ est un domaine fibré si $\partial M$ est l'union de deux surfaces compactes lisses $\partial_{h} M$ et $\partial_{v} M$ satisfaisant aux conditions suivantes :

- $\partial_{h} M$ est transversale au feuilletage $\tau$ tandis que $\partial_{v} M$ est tangente à $\tau$;

- les surfaces $\partial_{h} M$ et $\partial_{v} M$ ont le même bord - qui est la partie anguleuse de $\partial M$ et chaque composante $S$ de $\partial_{v} M$ a sur son bord des points où $M$ est concave (c'est-à-dire modelé non pas sur un quart mais sur trois quarts d'espace) ; 
- chaque feuille de $\tau$ traverse au plus deux fois $\partial_{v} M$.

Proposition 1.7. Le quotient $X=M / \tau$ est naturellement une surface branchée sans bord dont le lieu singulier est l'image des composantes $S$ de $\partial_{v} M$ telles que $M$ soit concave près de chaque point de $\partial S$.

Démonstration. Soit $\pi$ la projection $M \rightarrow X=M / \tau$. Pour tout point $p \in X$ situé hors de $\pi\left(\partial_{v} M\right)$, on prend comme carte locale une transversale à $\tau$. Si $p \in \pi\left(\partial_{v} M\right)$ est dans l'image d'une seule composante, on a deux cartes locales naturelles qui consistent à prendre des transversales à $\tau$ le long d'une composante du bord ou le long de l'autre.

Lorsque les deux premières conditions sont réalisées, on peut toujours obtenir la troisième, quitte à perturber $\tau$ dans l'intérieur de $M$.

On observe que chaque composante connexe de $\partial_{v} M$ est feuilletée par des intervalles compacts transversaux au bord et est donc un anneau.

Dès que $X$ a des points triples, il n'existe aucun plongement $\phi: X \rightarrow M$ qui soit une section de la projection $\pi: M \rightarrow X=M / \tau$. On peut cependant trouver des plongements $\left.\left.\phi_{s}: X \rightarrow M, s \in\right] 0,1\right]$, transversaux à $\tau$ qui sont des sections de $\pi$ en dehors du $s$-voisinage du lieu singulier et ont la propriété que $\pi \circ \phi_{s}$ converge vers l'identité uniformément quand $s$ tend vers 0 . On dit alors souvent, pour $s$ petit, que $M$ est un « voisinage fibré $»$ de $\phi_{s}(X)$.

Une surface $S \subset(M, \tau)$ est portée par $(M, \tau)$ si elle est transversale à $\tau$. Elle est pleinement portée lorsque de surcroît elle rencontre toutes les fibres de $\tau$.

\section{Structures de contact et triangulations}

Le but de cette partie est la démonstration du résultat suivant :

Théorème 2.1. Soit $V$ une variété close et orientée de dimension 3 . Il existe dans $V$ un nombre fini de domaines fibrés $\left(M_{i}, \tau_{i}\right)$ flanqués chacun d'une structure de contact $\zeta_{i}$ sur $V \backslash \operatorname{Int}\left(M_{i}\right)$ tels que toute structure de contact tendue sur $V$, à isotopie près et pour un certain $i$, soit égale à $\zeta_{i}$ hors de $M_{i}$ et tangente à $\tau_{i}$ dans $M_{i}$.

\subsection{Triangulations de contact}

Soit $V$ une variété de dimension 3. Ce qu'on appelle triangulation de $V$, dans la suite, est une décomposition simpliciale possédant les propriétés de régularité suivantes :

- chaque simplexe de dimension 2 (ou moins) est lisse ;

- chaque simplexe de dimension 3 a, le long de ses arêtes mais en dehors des sommets, un angle d'ouverture strictement compris entre 0 et $\pi$.

Si $\Delta$ est une triangulation de $V$, on note $\Delta^{i}, 0 \leq i \leq 3$, son squelette de dimension $i$. En outre, une isotopie de triangulations est un chemin de triangulations. Une telle isotopie $\Delta_{t}$ se prolonge en une isotopie ambiante $\psi_{t}$ de $V$ qui n'est bien sûr pas lisse en général mais se compose d'homéomorphismes dont les restrictions à chaque 2-simplexe de $\Delta_{0}$ sont lisses. On écrira $\Delta_{t}=\psi_{t}\left(\Delta_{0}\right)$ pour dire que $\Delta_{t}$ est l'image de $\Delta_{0}$ par $\psi_{t}$.

Toute triangulation lisse - c'est-à-dire dont chaque simplexe est lisse [Wh] - est évidemment une triangulation au sens ci-dessus mais on aura besoin ici de triangulations qui ne sont pas lisses. 
Définition 2.2 Gi6]. Soit $(V, \xi)$ une variété de contact de dimension 3. Une triangulation de contact de $(V, \xi)$ est une triangulation de $V$ vérifiant les conditions suivantes (voir la définition 2.3 pour la condition de convexité relative) :

1. les 1-simplexes sont des arcs legendriens ;

2 . les 2 -simplexes sont $\xi$-convexes et relativement $\xi$-convexes ;

3. les 3-simplexes sont contenus dans des cartes de Darboux 2 .

Une telle triangulation n'est pas lisse car les arêtes issues d'un sommet $p$ quelconque sont toutes tangentes au même plan, à savoir le plan de contact $\xi_{p}$.

On appelle nombre de Thurston-Bennequin d'une triangulation de contact $\Delta$ de $(V, \xi)$ l'entier

$$
\operatorname{TB}(\Delta)=\operatorname{TB}(\Delta, \xi)=-\sum_{F} \operatorname{tb}(\partial F),
$$

la sommation se faisant sur tous les 2-simplexes $F$. Comme $\operatorname{tb}(\partial F) \leq-1$ pour tout $F$ (d'après l'inégalité de Bennequin), l'entier $\operatorname{TB}(\Delta)$ est positif et au moins égal au nombre de 2 -simplexes de $\Delta$.

Une triangulation de contact $\Delta$ de $(V, \xi)$ sera dite minimale si elle a le plus petit nombre de Thurston-Bennequin parmi les triangulations de contact obtenues en déformant $\Delta$ par une isotopie à support compact et relative à un voisinage des sommets.

On explicite maintenant la condition technique de $\xi$-convexité relative qui, en pratique, est aussi facile à réaliser que la $\xi$-convexité ordinaire :

Définition 2.3. Soit $(V, \xi)$ une variété de contact et $\Delta$ une triangulation de $V$ dont le 1-squelette est legendrien. Soit $G$ un 3 -simplexe, $F_{0}, F_{1}$ deux faces de $G$ et $a$ leur arête commune. On oriente $F_{1}$ comme partie de $\partial G$ et $a$ comme partie de $\partial F_{1}$. On dira que $F_{0}$ et $F_{1}$ sont $\xi$-indisciplinées le long d'un arc (orienté) $[p, q] \subset a$ si $\xi$ est tangent à $F_{0}$ en $p$, à $F_{1}$ en $q$ et n'est un hyperplan d'appui de $G$ en aucun point de $] p, q 3^{3}$. On dit que les 2 -simplexes de $\Delta$ sont relativement $\xi$-convexes si aucun 3 -simplexe ne possède de faces $\xi$-indisciplinées le long d'un arc de leur arête commune.

On rappelle (cf. section 1.1) qu'un ensemble $\mathcal{X}$ de structures de contact tendues sur une variété $V$ close et orientée est dit complet s'il représente toutes les classes d'isotopie dans $\mathcal{S C T}(V)$. Le point de départ de cette étude est la proposition suivante, variante d'un résultat de [Gi6] servant à construire des livres ouverts adaptés aux structures de contact :

Proposition 2.4. Sur toute variété close et orientée $V$ de dimension 3 , il existe un ensemble complet $\mathcal{X}$ de structures de contact tendues et une triangulation $\Delta$ ayant les propriétés suivantes :

1. toutes les structures de contact de $\mathcal{X}$ coïncident - comme champs de plans non orientés - sur un voisinage des sommets de $\Delta$;

2. $\Delta$ est une triangulation de contact minimale de $(V, \xi)$ pour tout $\xi \in \mathcal{X}$.

\footnotetext{
${ }^{2}$ Cette condition est automatiquement remplie quand la structure de contact $\xi$ est tendue puisque, d'après El2, toute boule munie d'une structure de contact tendue se plonge dans l'espace de contact standard.

${ }^{3}$ Un tel arc $] p, q[$ est, en quelque sorte, une orbite de $\xi \partial G$ qui va d'une singularité négative à une singularité positive (voir la remarque [1.3).
} 
De plus, on peut choisir $\Delta$ isotope à n'importe quelle triangulation lisse fixée de $V$.

Avant de démontrer cette proposition, on traite le cas d'une seule structure de contact :

Lemme 2.5. Soit $(V, \xi)$ une variété de contact de dimension 3. Si la structure $\xi$ est tendue, toute triangulation lisse de $V$ est isotope à une triangulation de contact de $(V, \xi)$.

Démonstration. Soit $\Delta_{0}$ une triangulation lisse de $V$. La possibilité de déformer $\Delta_{0}$ par isotopie en une triangulation de contact $\Delta_{1}$ de $(V, \xi)$ tient avant tout au fait qu'on peut déformer chaque arête de $\Delta_{0}$ en un arc legendrien par une isotopie relative à ses extrémités - et arbitrairement $\mathcal{C}^{0}$-petite. Il faut toutefois un peu de soin aux sommets pour garantir la différentiabilité du prolongement aux 2-simplexes.

En chaque sommet $s$, on se donne une projection $d \pi_{s}: T_{s} V \rightarrow \xi_{s}$ dont le noyau est transversal aux plans tangents des 2-simplexes en $s$ et qui envoie les tangentes aux arêtes sur des droites distinctes. Sur un voisinage compact convenable $N(s)$, il existe des coordonnées $(x, y, z) \in \mathbf{D}^{2} \times[-1,1]$ centrées en $s$ dans lesquelles $d \pi_{s}$ est la dérivée de la projection $\pi_{s}:(x, y, z) \mapsto(x, y)$ et $\xi$ a pour équation $d z+x d y-y d x=0$. Par ailleurs, pour $N(s)$ assez petit, $\pi_{s}$ induit un plongement sur le 1-squelette et sur chaque 2-simplexe de $\Delta_{0}$. On déforme $\Delta_{0}$ dans $N(s)$ comme suit (voir l'exemple 2.6) :

- les arêtes de $\Delta_{1}$ sont les arcs legendriens sur lesquels les arêtes de $\Delta_{0}$ se projettent ;

- les faces de $\Delta_{1}$ sont des graphes au-dessus ou en-dessous de celles de $\Delta_{0}$;

- les arêtes et les faces des triangulations intermédiaires $\Delta_{t}$, pour tout $t \in[0,1]$, sont obtenues en prenant l'isotopie barycentrique verticale au temps $t$ entre les arêtes et les faces de $\Delta_{0}$ et celles de $\Delta_{1}$.

Moyennant quelques petites précautions dans la mise en place des 2 -simplexes de $\Delta_{1}$, les angles diédraux de ses 3-simplexes dans $N(s)$ sont compris strictement entre 0 et $\pi$ en dehors de $s$ et il en va alors de même pour les angles diédraux des 3-simplexes de chaque $\Delta_{t}, t \in[0,1]$. On a ainsi fabriqué les triangulations $\Delta_{t}$ voulues près des sommets.

On choisit ensuite une isotopie $\Delta_{t}^{1}$ du 1-squelette de $\Delta_{0}$ qui prolonge celle construite dans les cartes $N(s)$, est lisse en dehors et aboutit à un graphe legendrien $\Delta_{1}^{1}$. Dans $V^{\prime}=V \backslash \bigcup \operatorname{Int}(N(s))$, cette isotopie s'étend en une isotopie ambiante lisse $\psi_{t}$ engendrée par un champ qui, sur le bord latéral $\partial_{\mid} N(s)=\left(\partial \mathbf{D}^{2}\right) \times[-1,1]$ de chacun des voisinages $N(s)=\mathbf{D}^{2} \times[-1,1]$, est un multiple du champ $\partial_{z}$. Les images $\psi_{t}\left(\Delta_{0}^{2} \cap V^{\prime}\right)$ du 2-squelette ne se recollent pas a priori aux 2-simplexes bâtis dans $N(s)$ mais les arcs qu'ils tracent sur les anneaux $\partial_{\mid} N(s)$ sont des graphes au-dessus de ceux tracés par les 2-simplexes dans $N(s)$ (pour la projection $\left.\pi_{s}\right)$. Une déformation de $\psi_{t}\left(\Delta_{0}^{2} \cap V^{\prime}\right)$ près des anneaux $\partial_{\mid} N(s)$ et laissant fixe le 1-squelette $\Delta_{t}^{1}$ permet alors de le recoller aux 2-simplexes de $\Delta_{t}$ dans $N(s)$. On a ainsi modifié $\Delta_{0}$, par l'isotopie $\Delta_{t}, t \in[0,1]$, en une triangulation $\Delta_{1}$ dont les 1-simplexes sont des arcs legendriens. De plus, comme $\xi$ est tendue, les 3 -simplexes sont tous inclus dans des cartes de Darboux.

Quitte à décroître le nombre de Thurston-Bennequin du bord des 2-simplexes de $\Delta_{1}$ (par stabilisation), on peut, dans la construction précédente, choisir l'isotopie de graphes $\Delta_{t}^{1}$ de sorte que, le long de chaque arête de $\Delta_{1}$, l'enroulement de $\xi$ autour d'une face quelconque soit négatif ou nul. Ceci permet de déformer $\Delta_{1}$, par une isotopie ambiante lisse de $V$ relative au 1-squelette et à un voisinage des sommets, pour que $\xi$ ne fasse aucun demi-tour inversé par rapport à une face le long d'une arête de son bord. Par le même principe, on élimine tous les arcs d'arêtes le long desquels deux faces adjacentes sont $\xi$-indisciplinées. Les 2 -simplexes sont alors relativement $\xi$-convexes et on les rend $\xi$-convexes par une ultime petite perturbation de $\Delta_{1}$ - toujours stationnaire sur le 1squelette et un voisinage des sommets. On obtient ainsi une triangulation de contact $\Delta_{2}$ 
de $(V, \xi)$ isotope à $\Delta_{0}$. Enfin, puisque les triangulations de contact de $(V, \xi)$ qui sont isotopes à $\Delta_{2}$ relativement à un voisinage des sommets ont un nombre de ThurstonBennequin minoré, l'une d'elles est minimale.

Exemple 2.6. Si une face $F_{0}$ de la triangulation initiale $\Delta_{0}$ se projette sur le quadrant $Q=\{x \geq 0, y \geq 0\}$ - ce qui est toujours le cas quitte à prendre d'autres coordonnées de Darboux -, la face $F_{1}$ correspondante dans la triangulation de contact $\Delta_{1}$ a une équation du type $z=x y u(x, y)$ et son point complexe à l'origine est hyperbolique si $|u(0,0)|>1$ et elliptique si $|u(0,0)|<1$. On ne peut donc pas choisir arbitrairement la nature des points complexes que les faces de $\Delta_{1}$ présentent en leurs sommets (si deux arêtes de $\Delta_{0}$ issues de l'origine se projettent par exemple à l'intérieur du quadrant $Q$ et se trouvent de part et d'autre de $F_{0}$, le point complexe de $F_{1}$ ne peut être hyperbolique) mais on peut faire en sorte qu'ils soient tous elliptiques.

Démonstration de la proposition 2.4. Soit $\xi_{0}$ une structure de contact tendue et $\Delta_{0}$ une triangulation de contact minimale de $\left(V, \xi_{0}\right)$ (lemme 2.5). Soit $N=\bigcup N(s)$ un voisinage compact du 0-squelette de $\Delta_{0}$ dont chaque composante connexe est une carte de Darboux $N(s)=\mathbf{D}^{2} \times[-1,1]$ autour d'un sommet $s$. On prend les domaines $N(s)$ assez petits pour que leur intersection avec chaque 2-simplexe de $\Delta_{0}$ soit un graphe au-dessus de sa projection sur $\mathbf{D}^{2}$ et évite $\mathbf{D}^{2} \times\{ \pm 1\}$. Toute structure de contact sur $V$ est isotope à une structure $\xi$ qui coïncide avec $\xi_{0}$ sur $N$. Pour peu que $\xi$ soit tendue, le lemme 2.5 (ou plus exactement sa preuve) montre que $(V, \xi)$ possède une triangulation de contact (minimale) $\Delta$ isotope à $\Delta_{0}$ relativement à $N$. Par une isotopie lisse $\psi_{t}$ de $V$ stationnaire sur $N$, on déforme $\Delta_{0}$ en une triangulation $\Delta_{1}=\psi_{1}\left(\Delta_{0}\right)$ qui a le même 1 -squelette que $\Delta$ et des 2 -simplexes tous $\xi$-convexes 4 et relativement $\xi$-convexes (voir la preuve du lemme 2.5). Comme $\xi$ est tendue, $\Delta_{1}$ est une triangulation de contact minimale de $(V, \xi)$. Par conséquent, $\Delta_{0}$ est une triangulaton de contact minimale de $\left(V, \psi_{1}^{*} \xi\right)$, ce qui établit la proposition.

En pratique, les triangulations de contact minimales s'avèrent trop rigides. Il est notamment difficile d'appliquer aux 2-simplexes les lemmes de réalisation de feuilletages caractéristiques sans créer d'intersections indésirables entre eux près des sommets. Pour cette raison, on introduit maintenant une notion de triangulations de contact « maniables » pour laquelle on donne une variante de la proposition 2.4 .

Définition 2.7. Soit $(V, \xi)$ une variété de contact de dimension 3. Une triangulation de contact $\Delta$ de $(V, \xi)$ est dite maniable si chaque simplexe $F$ de dimension 2 écorné de trois triangles aux sommets est un hexagone $H_{F}$ à bord legendrien et $\xi$-convexe, et si, pour toute arête $a$, l'enroulement de $\xi$ autour de $F$ le long de l'arc $r(a)=a \backslash \operatorname{Int}(\Lambda)$, $\Lambda=\bigcup_{F} \overline{\left(F \backslash H_{F}\right)}$, est strictement négatif. (Noter que cet arc n'est, en général, qu'un morceau d'arête de $H_{F}$ mais pas une arête entière.) Dans le 2-squelette de $\Delta$, l'ensemble $\Lambda=\bigcup_{F} \overline{\left(F \backslash H_{F}\right)}$ est un voisinage des sommets qu'on nomme voisinage de sécurité. On dit que $\Delta$ est $\Lambda$-minimale si elle a le plus petit nombre de Thurston-Bennequin parmi toutes les triangulations de contact maniables de $(V, \xi)$ ayant $\Lambda$ pour voisinage de sécurité. Dit autrement en faisant porter la déformation sur $\xi$, la triangulation $\Delta$ est $\Lambda$-minimale si pour toute structure $\xi^{\prime}$ isotope à $\xi$ relativement à $\Lambda$ et pour laquelle $\Delta$ est une triangulation de contact maniable, $\operatorname{TB}\left(\Delta, \xi^{\prime}\right) \geq \operatorname{TB}(\Delta, \xi)$. On note $\Lambda_{F}(s)$ le triangle

\footnotetext{
${ }^{4}$ On utilise ici le fait que la $\xi$-convexité est une propriété à la fois dense et ouverte.
} 
de $\Lambda$ inclus dans la face $F$ et contenant le sommet $s$, et $\Lambda(s)$ la réunion des triangles de $\Lambda$ contenant $s$.

Proposition 2.8. Sur toute variété close et orientée $V$ de dimension 3 , il existe un ensemble complet $\mathcal{X}$ de structures de contact tendues et une triangulation $\Delta$ ayant les propriétés suivantes :

- toutes les structures de contact de $\mathcal{X}$ coïncident - comme champs de plans non orientés - sur un voisinage $U$ des sommets de $\Delta$;

- $\Delta$ est, pour tout $\xi \in \mathcal{X}$, une triangulation de contact maniable de $(V, \xi)$ dont le voisinage de sécurité $\Lambda$ est fixe et contenu dans $U$ et qui est $\Lambda$-minimale.

Démonstration de la proposition 2.8. Soit $\xi_{0}$ une structure de contact tendue et $\Delta$ une triangulation de contact de $\left(V, \xi_{0}\right)$ (le lemme 2.5 montre qu'il en existe). On reprend les notations de la preuve du lemme 2.5. Dans chaque petite boule $N(s)$ centrée en un sommet $s$ de $\Delta$, on décroît localement, par une isotopie de stabilisation, le nombre de ThurstonBennequin de chaque arête $a$ issue de $s$ afin que, dans $N(s)$, celui-ci soit strictement négatif et que $\xi_{0}$ imprime dans $N(s)$ un point singulier sur toute face adjacente à $a$. Dans cette situation, on peut tracer sur chaque face $F$ de sommet $s$ un arc $\gamma_{F, s}$ inclus dans $F \cap N(s)$, tangent à $\xi$ en ses extrémités et délimitant un triangle $\Lambda_{F}(s)$ dans $F$. Par approximation legendrienne, on est alors en mesure d'effectuer une isotopie de $\xi_{0}$ à support dans un voisinage de $\bigcup_{F, s} \gamma_{F, s}$ et relative au 1-squelette de $\Delta$ pour que $\gamma_{F, s}$ devienne un arc legendrien d'invariant de Thurston-Bennequin relatif à $F$ inférieur à -1 . La réunion des $\operatorname{arcs} \gamma_{F, s}$ découpe les arêtes de $\Delta$ en un certain nombre d'arcs legendriens. Quitte à stabiliser chacun de ces arcs, on se ramène au cas où, pour toute face $F$, ils ont tous un nombre de Thurston-Bennequin relatif à $F$ inférieur à -1 . Une isotopie à support dans un voisinage du 1-squelette permet ensuite d'éviter tous les demi-tours inversés le long des arêtes ainsi que des arcs $\gamma_{F, s}$. On rend alors les faces $\xi_{0}$-convexes et relativement $\xi_{0}$-convexes à l'aide d'une isotopie relative à $\Delta^{1}$ et aux $\operatorname{arcs} \gamma_{F, s}$. La triangulation $\Delta$ est de contact et maniable pour $\left(V, \xi_{0}\right)$ relativement au voisinage de sécurité $\Lambda=\bigcup_{F, s} \Lambda_{F}(s)$.

La proposition 2.4 permet maintenant d'isotoper toute structure $\xi$ sur une structure $\xi^{\prime}$ qui coïncide avec $\xi_{0}$ le long de $\Lambda$ et qui a $\Delta$ pour triangulation de contact. Le procédé de stabilisation fournit une isotopie de $\xi^{\prime}$ relative à $\Lambda$ qui donne aux trois côtés de l'hexagone $H_{F}$ inclus dans $\partial F$ des nombres de Thurston-Bennequin relatifs inférieurs à -1 . On fait même en sorte que les arcs $r(a)=\overline{a \backslash \Lambda} \subset a$ aient un invariant de Thurston-Bennequin

relatif à toute face adjacente inférieur à $-\frac{1}{2}$. La triangulation $\Delta$ est donc maniable pour $\xi^{\prime}$ et a $\Lambda$ comme voisinage sûr. Comme dans le lemme 2.5, on peut également la supposer $\Lambda$-minimale. L'ensemble $\mathcal{X}$ est constitué du choix d'une structure $\Lambda$-minimale pour $\Delta$ dans chaque classe d'isotopie de structures tendues.

Dans toute la suite du texte, on désigne par $\mathcal{X}$ un système complet de structures de contact tendues sur $V$ et par $\Delta$ une triangulation de $V$ tels que $\Delta$ soit une triangulation de contact maniable et $\Lambda$-minimale pour toute structure $\xi \in \mathcal{X}$, où $\Lambda$ est un voisinage de sécurité fixe le long duquel toutes les structures de $\mathcal{X}$ coïncident.

\subsection{Propriétés des triangulations de contact minimales}

On commence par une propriété valable pour toutes les triangulations de contact.

Lemme 2.9. Si $\Delta$ est une triangulation de contact pour $(V, \xi)$, pour toute face $F \in \Delta^{2}$, aucune composante de la courbe de découpage $\Gamma_{F}(\xi)$ n'est un cercle. 
Démonstration. Cette absence de courbes fermées vaut en fait pour le découpage de toute surface $\xi$-convexe autre qu'une sphère et au voisinage de laquelle $\xi$ est tendue. Or chaque 3 -simplexe de $\Delta$ est inclus dans une carte de Darboux donc, a fortiori, $\xi$ est tendue au voisinage de $F$. (Si une composante de $\Gamma_{F}$ était un cercle, le lemme de réalisation de feuilletages permettrait de faire apparaître un disque vrillé.)

Soit $(V, \xi)$ une variété de contact tendue de dimension 3 munie d'une triangulation de contact $\Delta$ maniable et $\Lambda$-minimale, où $\Lambda$ est son voisinage de sécurité. Pour une arête $a$ de $\Delta$, on rappelle que $r(a)=\overline{a \backslash \Lambda}$ et que $H_{F}$ est l'hexagone $F \backslash\left(\bigcup_{1 \leq i \leq 3} \operatorname{Int}\left(\Lambda_{F}\left(s_{i}\right)\right)\right)$.

Soit $F$ une face de $\Delta$. On dira qu'une composante $\Gamma$ de $\Gamma_{H_{F}}(\xi)$ est extrémale si un des deux points $p$ de $\partial \Gamma$ appartient à un arc $r(a)$, où $a$ est une arête de $F$, ne peut pas être poussé en dehors de $r(a)$ par une isotopie de $\Gamma$ parmi les courbes transversales à $\xi F$ (autrement dit il y a des singularités de $\xi F$ entre $p$ et les extrémités de $r(a)$ ) et est le plus proche d'une des extrémités de $r(a)$ parmi les points de $\Gamma_{H_{F}}(\xi) \cap r(a)$ qui possèdent ces propriétés. En particulier, pour un certain choix de $\Gamma_{H_{F}}(\xi)$, un point de $\Gamma \cap r(a)$ est effectivement extrême. La multicourbe $\Gamma_{H_{F}}(\xi)$ contient au plus six composantes extrémales.

Lemme 2.10. Si $\xi$ est une structure de contact tendue sur $V$ et si $\Delta$ est une triangulation de contact maniable et $\Lambda$-minimale pour $\xi$, alors toute composante du découpage $\Gamma_{H_{F}}(\xi)$ parallèle à $r(a)$ est extrémale.

Démonstration. On suppose qu'une composante de $\Gamma_{H_{F}}(\xi)$ parallèle à $r(a)$ n'est pas extrémale. Ceci signifie en particulier que l'enroulement de $\xi$ le long de $r(a)$ relativement à $F$ est inférieur à -2 . Le lemme de réalisation 1.6 permet, par une isotopie de $H_{F}$ relative à un voisinage de $\partial H_{F} \backslash r(a)$ et de support inclus dans un petit voisinage homogène de $H_{F}$, de déformer $H_{F}$ en une surface $H_{F}^{\prime}$ qui contient une rocade $D$ s'appuyant sur $r(a)$ (c'est-à-dire que $\partial_{-} D \subset r(a)$ et $\operatorname{Int}\left(\partial_{\cap} D\right) \subset \operatorname{Int}\left(H_{F}^{\prime}\right)$ ). La surface $\overline{H_{F} \backslash D}$, une fois les coins de $D$ lissés, est un hexagone $H_{F}^{\prime}$ à bord legendrien qui est $\xi$-convexe et isotope à $H_{F}$. Par construction, $t b\left(H_{F}^{\prime}\right)=t b\left(H_{F}\right)+1$. L'isotopie entre $H_{F}$ et $H_{F}^{\prime}$ se prolonge en une isotopie $\left(\phi_{t}\right)_{t \in[0,1]}$ de $V$ à support dans un voisinage de $D$ et donc stationnaire sur $\Lambda$. Le 1-squelette de $\Delta$ est legendrien pour $\xi^{\prime}=\phi_{1}^{*} \xi$ et l'arc $r(a)$ possède un nombre de Thurston-Bennequin relatif aux faces adjacentes inférieur ou égal à $-\frac{1}{2}$ (et même à -1 relativement à $F$ ) et strictement supérieur à celui donné par $\xi$. Une isotopie de $\xi^{\prime}$ relative au 1-squelette de $\Delta$ et à $\Lambda$ permet de rendre les faces $\xi^{\prime}$-convexes et relativement $\xi^{\prime}$-convexes. Elle fait de $\Delta$ une triangulation de contact maniable pour la nouvelle structure $\xi^{\prime \prime}$. Ce faisant, on a strictement diminué le nombre de Thurston-Bennequin et donc $\Delta$ n'était pas $\Lambda$-minimale pour $\xi$.

Si $F$ est un 2-simplexe de $\Delta$, un quadrilatère fibré dans $F$ est un quadrilatère $[0,1] \times[0,1] \subset F$ dont l'intersection avec $\partial F$ est l'union des deux arêtes verticales $\{0\} \times[0,1]$ et $\{1\} \times[0,1]$, celles-ci se trouvant à l'intérieur de deux arêtes distinctes de $F$. Un arc simple et propre $A \subset F$ est porté par un quadrilatère fibré $Q$ s'il est inclus dans $Q \backslash\{y=0,1\}$ et s'il est transversal au champ de vecteurs $\partial_{y}$.

Soit $(V, \xi)$ une variété de contact tendue de dimension 3 et $\Delta$ une triangulation de contact de $(V, \xi)$. Pour toute face $F$ de $\Delta$, on note $\Gamma_{F}$ le découpage de $\xi F$ associé à un épaississement homogène quelconque de $F$. On appelle pièce de $F$ l'adhérence de toute composante connexe de $F \backslash \Gamma_{F}$ et on dit qu'une pièce est ordinaire ou extraordinaire selon que c'est ou non un quadrilatère fibré. On insiste ici sur le fait qu'on ne fixe pas l'épaississement homogène une fois pour toutes et qu'on s'autorise donc des isotopies de $\Gamma_{F}$ parmi les multi-courbes transversales à $\xi F$. 
Corollaire 2.11. Soit $\mathcal{X}$ un système complet de structures de contact tendues sur $V$ et $\Delta$ une triangulation de $V$ qui est maniable et $\Lambda$-minimale pour tout $\xi \in \mathcal{X}$, avec un voisinage de sécurité $\Lambda$ fixe le long duquel toutes les structures de $\mathcal{X}$ coïncident. Il existe $C_{0}>0$ tel que, pour tout $\xi \in \mathcal{X}$, toute face $F$ de $\Delta$ contienne trois quadrilatères fibrés $Q_{1}, Q_{2}, Q_{3}$ deux à deux disjoints, s'appuyant sur des paires d'arêtes de $F$ distinctes et inclus dans $H_{F} \backslash \Lambda$ (en particulier, pour $i \in\{1,2,3\}$, les arêtes verticales de $Q_{i}$ sont incluses dans $\left.\bigcup_{a \subset \Delta^{1}} r(a)\right)$, avec les propriétés suivantes :

- chaque $Q_{i}$ est une union de pièces ordinaires ;

- au plus $C_{0}$ pièces du découpage de $\xi F$ ne sont pas incluses dans $Q_{1} \cup Q_{2} \cup Q_{3}$.

Démonstration. Il découle du lemme 2.10 que les pièces ordinaires qui n'intersectent pas $\Lambda$ forment trois quadrilatères fibrés « non parallèles ». Vu le lemme 2.9, restent comme pièces au plus six demi-disques extrémaux, une pièce « centrale », et des pièces qui rencontrent $\Lambda$ et dont le nombre est donc borné indépendemment de $\xi \in \mathcal{X}$ (voir la figure (2).

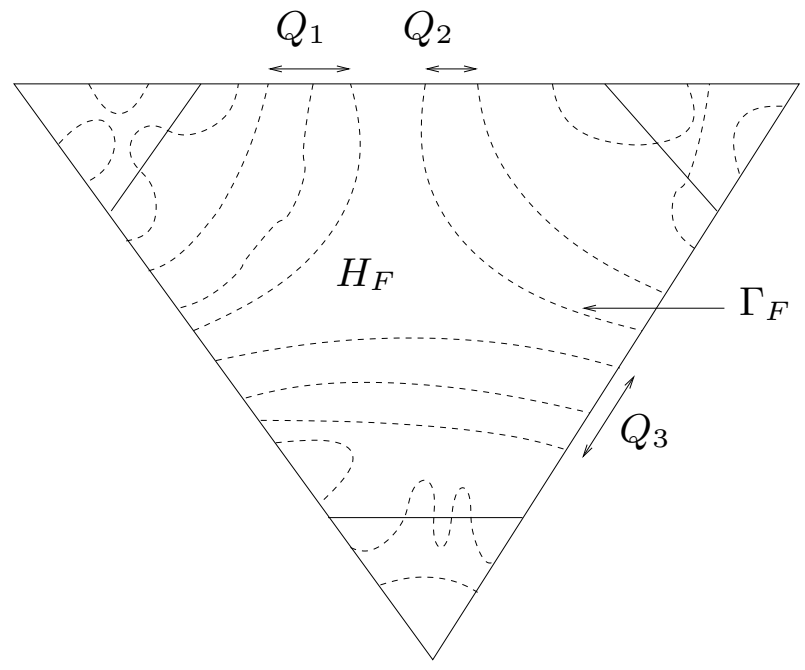

Fig. 2 - Le découpage de $F$ et $H_{F}$.

\subsection{Prismes fibrés}

Soit $Y$ un triangle ou un quadrilatère. Un prisme fibré est un polyèdre $P=Y \times[0,1]$ dont on ne retient de la structure produit que la projection sur $Y$. Les faces de $P$ sont dites verticales ou horizontales selon qu'elles se trouvent dans $\partial Y \times[0,1]$ ou dans $Y \times\{0,1\}$.

Soit $\Delta$ une triangulation de $V$. Un prisme fibré dans $(V, \Delta)$ est un plongement d'un prisme fibré $P$ dans $V$ avec les propriétés suivantes:

- $P$ est contenu dans un 3-simplexe $G$ de $\Delta$ et son intersection avec $\partial G$ est l'union de ses faces verticales ;

- chaque face verticale de $P$ est un quadrilatère fibré d'une face de $G$.

Un 3-simplexe donné contient au plus cinq prismes fibrés deux à deux disjoints et non isotopes parmi les prismes fibrés. Il y a, à isotopie près parmi les prismes fibrés, trois telles familles de cinq prismes fibrés : chacune d'elle possède quatre prismes à base triangulaire, situés près des sommets, et un prisme de base quadrilatérale, situé en diagonale. 
Une configuration de prismes fibrés dans $(V, \Delta)$ est la donnée d'une collection de prismes fibrés $P=\left(P_{i}\right)_{1 \leq i \leq k}$ de $(V, \Delta)$ qui intersecte chaque 3 -simplexe de $\Delta$ en une sous-famille de l'une des trois familles maximales de cinq prismes précédentes et telle que l'intersection entre deux prismes $P_{i} \neq P_{j}$ de $P$ donne soit un quadrilatère fibré (avec concordance des fibrations données de part et d'autre), soit un arc d'intérieur non vide inclus dans une arête de $\Delta$, soit l'ensemble vide. Dans le premier cas, les prismes $P_{i}$ et $P_{j}$ sont contenus dans des 3-simplexes qui ont une face en commun et dans le deuxième cas, dans des 3-simplexes qui ont une arête commune.

Lemme 2.12. Toute variété triangulée $(V, \Delta)$ possède un nombre fini de configurations de prismes fibrés, à isotopie près parmi les configurations de prismes fibrés.

Une configuration de prismes fibrés $P$ est dite admissible pour $\xi \in \mathcal{X}$ si les faces verticales des prismes de la famille $P$ sont des unions de pièces ordinaires qui ne rencontrent pas $\Lambda$. Pour $\xi \in \mathcal{X}$, on note $\mathbf{P}_{\Delta, \xi}$ l'ensemble des configurations de prismes fibrés admissibles pour $\xi$. On munit les classes d'isotopies de prismes fibrés dans $\mathbf{P}_{\Delta, \xi}$ d'une relation d'ordre partiel : si $P, Q \in \mathbf{P}_{\Delta, \xi}$ représentent des classes d'isotopies $[P]$ et $[Q]$, on dit que $[P] \preceq[Q]$ s'il existe une isotopie de $P$ dans $\mathbf{P}_{\Delta, \xi}$ en $P^{\prime} \subset Q$.

Lemme 2.13. Pour $\xi \in \mathcal{X}$ fixée, les classes d'isotopie de $\mathbf{P}_{\Delta, \xi}$ sont en nombre fini.

Démonstration. Il suffit d'observer qu'un élément de $\mathbf{P}_{\Delta, \xi}$ est déterminé, à isotopie près dans $\mathbf{P}_{\Delta, \xi}$, par la classe d'isotopie de ses arêtes horizontales parmi les arcs transversaux aux feuilletages caractéristiques des faces. Or toute arête horizontale incluse dans une face $F$ est isotope à une composante de $\Gamma_{F}(\xi)$, ce qui donne un nombre fini de classes d'isotopie possibles pour chaque arête horizontale, mais également pour leur collection.

Les sous-sections suivantes sont consacrées à la démonstration du lemme fondamental ci-dessous :

Lemme 2.14. Soit $\mathcal{X}$ un ensemble complet de structures de contact tendues sur $V$ et $\Delta$ une triangulation de $V$ qui est maniable et $\Lambda$-minimale pour tout $\xi \in \mathcal{X}$, avec un voisinage de sécurité $\Lambda$ fixe le long duquel toutes les structures de $\mathcal{X}$ coïncident. Il existe $C_{1}>0$ tel que pour tout $\xi \in \mathcal{X}$, et pour tout élément $P=\left(P_{i}\right)_{1 \leq i \leq n}$ de $\mathbf{P}_{\Delta, \xi}$ dont la classe d'isotopie est maximale, au plus $C_{1}$ pièces des faces de $\Delta$ ne sont pas incluses dans $\operatorname{Int}\left(\bigcup_{1 \leq i \leq n} P_{i}\right)$.

Remarque 2.15. Quitte à considérer des sous-familles de prismes $P$ des éléments maximaux de $\mathbf{P}_{\Delta, \xi}$ et à augmenter la valeur de $C_{1}$, on peut également assurer que chaque face verticale des prismes de $P$ contient au moins 20 pièces (ordinaires).

\subsubsection{Une première normalisation des faces}

Pour toute arête $a$ de $\Delta$, on note $s(a)=\bigcup_{F \supset a}\left(a \cap H_{F}\right)$.

Lemme 2.16. Quitte, pour tout $\xi \in \mathcal{X}$, à effectuer une isotopie de $\xi$, on peut supposer que, si $\xi \in \mathcal{X}$ :

1. $\Delta$ est une triangulation de contact maniable et $\Lambda$-minimale pour $\xi$;

2. pour toute arête $a \subset \Delta^{1}$, il existe un germe de feuilletage $\mathcal{F}_{a}$ au voisinage de $s(a)$ par des arcs parallèles à $s(a), s(a)$ étant une feuille, qui est tangent au germe des faces adjacentes à $s(a)$ et qui est legendrien pour tout $\xi \in \mathcal{X}$; 
3. pour toute face $F$ et pour toute pièce ordinaire $R$ incluse dans $F \backslash \Lambda$,

$$
\left.\xi\right|_{R}=\{\sin \theta d x+\cos \theta d y=0\}
$$

si $R \simeq\{y=0\} \subset\{(x, y, \theta) \in[0,1] \times[-1,1] \times[-\pi / 2, \pi / 2]\}$. En particulier, le feuilletage caractéristique de $R$ possède une courbe de singularités $\{y=0, \theta=0\}$ portée par $R$.

Démonstration. La deuxième condition n'est a priori pas réalisée dans le voisinage de sécurité $\Lambda$. Pour démontrer ce lemme, on reprend la démonstration de la proposition 2.8, dans laquelle on exige une propriété supplémentaire pour la structure $\xi_{0}$ : on déforme $\xi_{0}$ par isotopie à l'aide du lemme de Darboux pour obtenir le point 2. C'est possible sans stabilisation supplémentaire puisque tous les enroulements relatifs aux faces sont négatifs. On déroule la preuve de la proposition 2.8 pour cette structure $\xi_{0}$, dont on déduit la construction d'un système complet $\mathcal{X}$ convenable. Pour une structure $\xi \in \mathcal{X}$ quelconque, on a alors 2 au voisinage de $s(a) \cap \Lambda$ où $\xi=\xi_{0}$. Le lemme de Darboux, appliqué à $\xi \in \mathcal{X}$ au voisinage d'une arête $a$ relativement à ce qui a déjà été fait au voisinage de $\Lambda$, fournit une isotopie de $\xi$ stationnaire sur $\Lambda$ qui conduit à une structure, notée à nouveau $\xi$, qui possède également un feuilletage legendrien par des arcs parallèles à $s(a)$ et tangent aux faces près de $s(a)$.

Pour obtenir un germe de feuilletage legendrien indépendant de $\xi \in \mathcal{X}$, on se sert du fait que le nombre de Thurston-Bennequin relatif à toute face $F$ contenant $a$ le long de $r(a)$ est inférieur à $-\frac{1}{2}$, ce pour tout $\xi \in \mathcal{X}$. Précisément, sur un voisinage $K$ de $s(a), \xi$ est solution d'une équation $\cos f_{0}(x, y, \theta) d x-\sin f_{0}(x, y, \theta) d y=0$ dans des coordonnées $(x, y, \theta) \in D^{2} \times[0,1], s(a)=\{x=y=0\}$, données par un plongement $\phi_{0}: D^{2} \times[0,1] \rightarrow V$ et pour lesquelles les faces sont $\partial_{\theta}$-invariantes. On déforme le feuilletage legendrien dirigé par $\partial_{\theta}$ sur un petit voisinage de $s(a)$ dans $K$ relativement à $\partial K$ tout en préservant les faces, pour le faire coïncider avec le feuilletage donné par $\xi_{0}$ tout près de $s(a)$. On obtient ainsi une isotopie $\left(\phi_{t}\right)_{t \in[0,1]}$ de $\phi_{0}$ pour laquelle $\phi_{1 *} \partial_{\theta}$ est tangent à $\xi_{0}$ près de $s(a)$ et $\left.\phi_{t}\right|_{\partial K}=\left.\phi_{0}\right|_{\partial K}$ pour tout $t \in[0,1]$. À chaque instant $t \in[0,1]$, la structure $\phi_{t}^{*} \xi$ est donnée près de $D^{2} \times\{0,1\}$ par une équation $\cos f_{t}(x, y, i) d x-\sin f_{t}(x, y, i) d y=0, i=0,1$, où $f_{t}$ dépend continûment de $t \in[0,1]$. Le fait que l'enroulement de $\xi$ le long de $s(a)$ par rapport à une face quelconque d'arête $a$ soit inférieur à $-\frac{1}{2}$ implique que pour tout $t \in[0,1], f_{t}(x, y, 1)>f_{t}(x, y, 0)$. On peut donc étendre $f_{t}$ en une famille continue de fonctions sur $D^{2} \times[0,1]$ constamment égale à $f$ près du bord avec la propriété $\partial_{\theta} f_{t}>0$. L'équation $\cos f_{t}(x, y, \theta) d x-\sin f_{t}(x, y, \theta) d y=0$ définit une structure de contact sur $D^{2} \times[0,1]$, dont l'image par $\phi_{t}$ se recolle à $\xi$ hors de $K$ pour former un chemin de structures de contact entre $\xi$ et une structure $\xi^{\prime}$ tangente au même feuilletage legendrien que $\xi_{0}$ près des arêtes. Le théorème de Gray convertit ce chemin en une isotopie de $V$ stationnaire sur $(V \backslash K) \cup \Delta^{1}$.

On obtient 3 relativement à la déformation déjà effectuée près des arêtes à l'aide du lemme de réalisation de feuilletages.

Dans la suite, le système $\mathcal{X}$ qu'on considère possède, en plus des précédentes, les propriétés explicitées dans les conclusions du lemme 2.16.

\subsubsection{Holonomie}

Soit $G$ un 3-simplexe dans une variété de contact $(V, \xi)$. Un arc legendrien de classe $\mathcal{C}^{1}$ par morceaux $\gamma_{1}:[0,1] \rightarrow \partial G$ qui évite les sommets de $G$ est dit étal si le champ 
de plans $\xi$ est un plan d'appui à $\partial G$ le long de $\gamma_{1}$. Précisément, $\gamma_{1}$ intersecte l'intérieur de chaque face le long d'une ligne singulière de son feuilletage caractéristique et, le long d'une arête, $\xi$ est à l'« extérieur »de $G$.

Une courbe d'holonomie est une courbe legendrienne $\gamma$ dans $\partial G$ constituée de la concaténation de deux arcs $\gamma_{1}$ et $\gamma_{2}$, où $\gamma_{1}$ est étal et $\gamma_{2}$ inclus dans une arête de $G$. On appelle champ de plans médian de $G$ le long de $\gamma_{2}$, tout champ de plans tangent à $\gamma_{2}$ qui rencontre l'intérieur du secteur de $T_{\gamma_{2}} V$ délimité par $G$, c'est-à-dire qui n'est un plan d'appui en aucun point de $\gamma_{2}$. L'holonomie $\operatorname{Hol}(\gamma)$ d'une courbe d'holonomie $\gamma$ est un entier dont la valeur absolue est égale à celle du nombre de Thurston-Bennequin de $\xi$ le long de $\gamma_{2}$, calculé relativement à un champ de plans médian quelconque de $G$ donné le long de $\gamma_{2}$. La valeur absolue de l'holonomie est donc la moitié du nombre de points de $\gamma_{2}$, comptés algébriquement, où $\xi$ est égal au plan médian. Il est positif si $\gamma_{2}$ est orienté comme le bord de la face qui contient $\gamma_{1}$ près de $\gamma_{1}(0)$ et négatif sinon. La figure 3 montre un exemple de courbe d'holonomie -1. Sur cette figure, lorsqu'on change le sens de parcours de $\gamma$, on change également la face servant à étalonner l'orientation de $\gamma_{2}$ et le signe de $\operatorname{Hol}(\gamma)$ ne varie pas. C'est toujours le cas lorsque $\gamma_{1}$ aborde $\gamma_{2}$ par deux faces différentes.

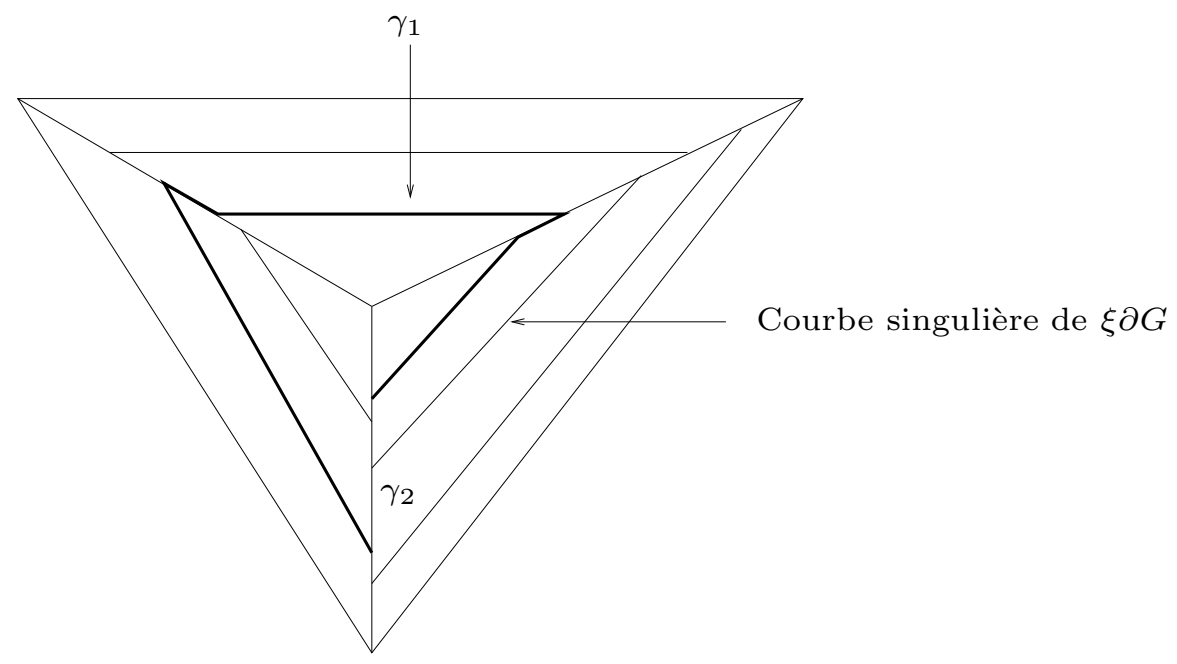

Fig. 3 - Courbe d'holonomie -1 .

Lemme 2.17. Soit $\xi \in \mathcal{X}$ et $G$ un 3-simplexe de $\Delta$. Toute courbe d'holonomie $\gamma$ pour $\xi$ incluse dans $\partial G \backslash \Lambda$ a une holonomie égale $\grave{a}-1$.

Remarque 2.18. Si $\gamma=\gamma_{1} \cup \gamma_{2}$ est une courbe d'holonomie pour laquelle $\gamma_{1}$ aborde $\gamma_{2}$ des deux bouts par la même face, et si $\bar{\gamma}$ est $\gamma$ orientée en sens inverse, alors $\operatorname{Hol}(\bar{\gamma})=-\operatorname{Hol}(\gamma)$. En particulier ce cas de figure est exclus par le lemme 2.17.

Démonstration. Comme $\xi$ est tendue, l'inégalité de Bennequin appliquée à $\gamma$ dit que $\operatorname{Hol}(\gamma) \neq 0$. On suppose que $\operatorname{Hol}(\gamma) \neq-1$. La courbe $\gamma$ se décompose par définition en la réunion de deux arcs $\xi$-legendriens $\gamma_{1}$ et $\gamma_{2}$, où $\gamma_{1}$ est étal et $\gamma_{2}$ est inclus dans un certain $r(a), a \in \Delta^{1}$. Comme $\gamma_{1}$ est étal, on peut le pousser sur un arc legendrien lisse $\gamma_{1}^{\prime}$ situé à l'intérieur de $G$ et s'appuyant sur $\partial \gamma_{2}$, via une famille d'arcs legendriens. En particulier, la courbe fermée $\gamma_{1}^{\prime} \cup \gamma_{2}$ borde un demi-disque $D$ avec $\operatorname{Int}(D) \subset \operatorname{Int}(G)$. Le nombre de Thurston-Bennequin de $\gamma_{2}$ relatif à $D$ vaut $-|H o l(\gamma)|$ si $\gamma_{1}$ aborde les deux 
bouts de $\gamma_{2}$ par le même côté, et sinon $-|\operatorname{Hol}(\gamma)|+\frac{1}{2} \operatorname{lorsque} \operatorname{Hol}(\gamma)<0$ et $-|\operatorname{Hol}(\gamma)|-\frac{1}{2}$ lorsque $\operatorname{Hol}(\gamma)>0$. En effet, cet enroulement est le même que celui de $\xi$ le long de $\gamma_{2}$, calculé par rapport à un champ de plans qui est médian de $G$ le long de $\operatorname{Int}\left(\gamma_{2}\right)$ et égal à $\xi$ (i.e. tangent à une face de $G$ ) aux points de $\partial \gamma_{2}$. Selon les cas, un tel champ de plans a un enroulement $0,-\frac{1}{2}$, où $\frac{1}{2}$ par rapport à un champ de plans médian. De la même façon, comme $\gamma_{1}$ est étal, l'invariant de Thurston-Bennequin de $\gamma_{1}^{\prime}$ relatif à $D$ vaut respectivement $0,-\frac{1}{2}$ et $\frac{1}{2}$ dans les trois cas énumérés ci-dessus. Dans toutes les situations, puisque $\operatorname{Hol}(\gamma) \neq-1$, on a $t b\left(\gamma_{1}^{\prime}, D\right) \geq t b\left(\gamma_{2}, D\right)+1$. On stabilise $\gamma_{1}^{\prime}$ pour obtenir un arc $\gamma_{1}^{\prime \prime}$, tel que $t b\left(\gamma_{1}^{\prime \prime}, D^{\prime}\right)=t b\left(\gamma_{2}, D^{\prime}\right)+1$ (si $D^{\prime} \subset G$ désigne l'image de $D$ par l'isotopie de stabilisation). Le disque $D^{\prime}$ va jouer le rôle de rocade.

L'isotopie qui consiste à pousser $\gamma_{2}$ sur $\gamma_{1}^{\prime \prime}$ le long de $D^{\prime}$ peut être réalisée relativement à $\Lambda$. Appliquée à $\xi$ elle fait de $\Delta$ une triangulation de contact pour son image $\xi^{\prime}$ (après déformations habituelles près des faces), avec un nombre de Thurston-Bennequin strictement moindre que celui de $\xi$. On constate de plus facilement que comme $\operatorname{Hol}(\gamma) \neq-1$, pour toute face $F$ de $\Delta$ et toute arête $a$ de $F$, le nombre de Thurston-Bennequin le long de $r(a)$ relatif à $F$ reste inférieur à $-\frac{1}{2}$. Cette triangulation est donc maniable pour $\xi^{\prime}$ et on obtient une contradiction avec la $\Lambda$-minimalité de $\Delta$.

\subsubsection{Démonstration du lemme 2.14}

Soit $G$ un 3 -simplexe de $\Delta$. On note $s_{1}, s_{2}, s_{3}$ et $s_{4}$ les sommets de $G$. La notation $\left[s_{i} s_{j}\right]$ désigne l'arête de $G$ qui joint les sommets $s_{i}$ et $s_{j}$. On note $\left(s_{i} s_{j} s_{k}\right)$ la face de $G$ qui contient les sommets $s_{i}, s_{j}$ et $s_{k}$.

Un paquet est une union de pièces ordinaires contenues dans une même face qui forme un quadrilatère fibré (connexe). L'épaisseur d'un paquet est le nombre de pièces qui le constituent.

Soit $\xi \in \mathcal{X}$ et $P=\left(P_{i}\right)_{1 \leq i \leq n} \in \mathbf{P}_{\Delta, \xi}$ une configuration de prismes admissible maximale. Les fonctions $f_{i}: \mathbb{R} \rightarrow \mathbb{R}$ qui apparaissent dans la suite sont affines, de dérivées strictement positives et indépendantes de $(\xi, P) \in \mathcal{X} \times \mathbf{P}_{\Delta, \xi}$. On raisonne par l'absurde en supposant que $N \gg 1$ pièces ne sont pas incluses dans $\operatorname{Int}\left(\bigcup_{1 \leq i \leq n} P_{i}\right)$. Parmi celles-ci au moins $N_{1}=f_{1}(N)$ pièces se trouvent dans une même face $F$ de $\Delta$. Le corollaire 2.11 nous dit alors qu'au moins $N_{2}=f_{2}\left(N_{1}\right)$ pièces ordinaires sont situées dans un même hexagone $H_{F} \backslash \Lambda$; leur union formant un paquet $Q_{1}$ (d'épaisseur $N_{2}$ ), lui-même contenu dans $H_{F} \backslash \operatorname{Int}_{G}\left(\left(\bigcup_{1<i<n} P_{i}\right) \cap G\right)$ pour un des deux simplexes $G$ de $\Delta$ contenant $F$. C'està-dire que $Q_{1}$ ne rencontre pas la réunion $P_{G}$ des prismes de $P$ inclus dans $G$. Soit $s_{1}, s_{2}$ et $s_{3}$ les sommets de $F$ et $s_{4}$ le quatrième sommet de $G$. Pour fixer les idées, disons que le paquet $Q_{1}$ joint $\left[s_{1} s_{2}\right]$ à $\left[s_{1} s_{3}\right]$. Au moins $N_{2}-1$ composantes de $\Gamma_{\left(s_{1} s_{3} s_{4}\right)}(\xi)$ partent de $\left[s_{1} s_{3}\right] \cap Q_{1}$, dont au moins $N_{3}=f_{3}\left(N_{2}\right)$ restent dans $H_{\left(s_{1} s_{3} s_{4}\right)} \backslash \Lambda$ et ne sont pas parallèles à une arête (corollaire 2.11).

Cas 1. Parmi celles-ci, au moins la moitié $N_{4}=f_{4}\left(N_{3}\right)$ vont vers $\left[s_{1} s_{4}\right]$ et délimitent un paquet $Q_{2}$ d'épaisseur $N_{4}-1$. Comme toutes ont une extrémité dans $\left[s_{1} s_{3}\right] \cap Q_{1}, Q_{2}$ ne rencontre pas $P_{G}$. On distingue à présent deux cas (figure 4) :

Cas 1a. Au moins 11 (pour être tranquille) composantes de $\Gamma_{H_{\left(s_{1} s_{2} s_{4}\right)}}(\xi)$ issues de $Q_{2} \cap\left[s_{1} s_{4}\right]$ reviennent vers $r\left(\left[s_{1} s_{2}\right]\right)$. On en déduit l'existence d'un paquet $Q_{3}$ d'épaisseur au moins 10 entre $\left[s_{1} s_{4}\right] \cap Q_{2}$ et $\left[s_{1} s_{2}\right]$ qui ne rencontre pas $P_{G} \cup \Lambda$.

Lemme 2.19. Il existe un prisme fibré $P^{\prime}$ dont le bord vertical est inclus dans $Q_{1} \cup Q_{2} \cup Q_{3}$ et est une union de pièces ordinaires. De plus, ce prisme ne rencontre pas $P_{G} \cup \Lambda$. 


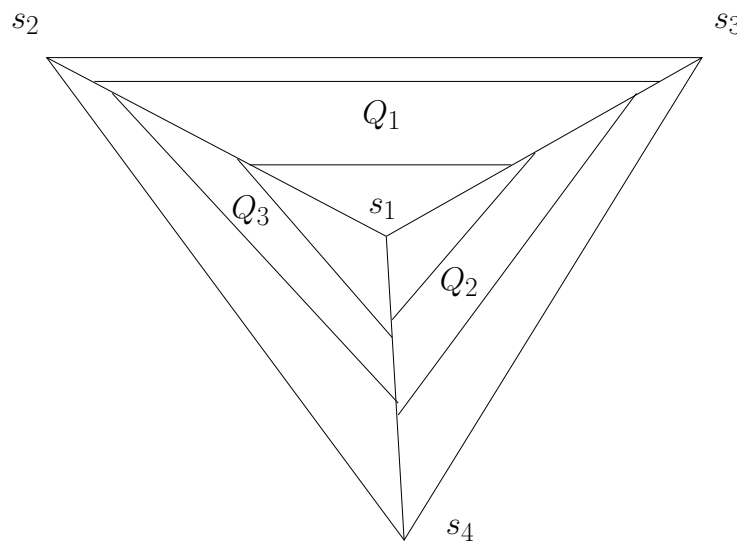

Le cas 1a.

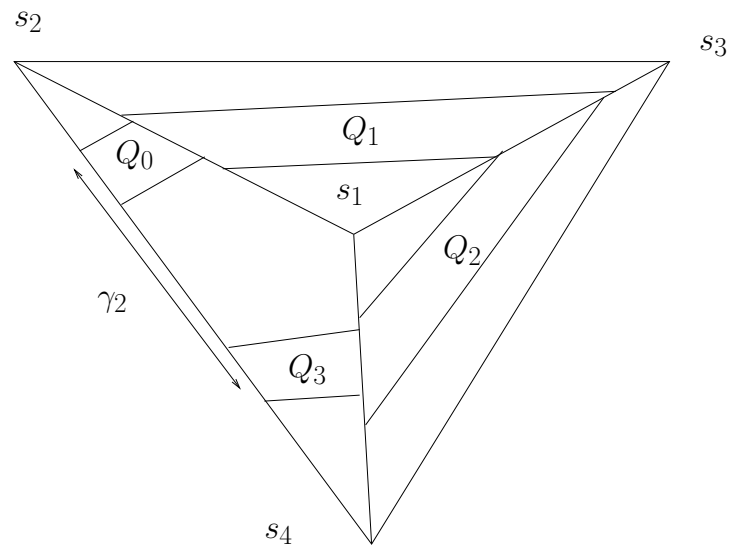

Le cas $1 b$.

FIG. 4 - Les cas $1 \mathrm{a}$ et $1 \mathrm{~b}$.

Démonstration. Il s'agit de construire les faces verticales de $P^{\prime}$. On ordonne les pièces contenues dans $Q_{3}$ de un à dix. Chaque pièce porte une courbe legendrienne singulière, numérotée comme la pièce dont elle fait partie. On part de la cinquième courbe singulière de $\xi Q_{3}$, notée $c_{3}$. L'extrémité de celle-ci dans $\left[s_{1} s_{4}\right]$ est encadrée par les extrémités de 2 courbes singulières de $\xi Q_{2}$. On note $c_{2}$, celle dont l'extrémité est située juste après $\partial c_{3} \cap\left[s_{1} s_{4}\right]$, pour l'orientation de $\left[s_{1} s_{4}\right]$ donnée comme bord de la face $\left(s_{1} s_{2} s_{4}\right)$. En particulier, la portion d'arête de $\left[s_{1} s_{4}\right]$ située entre $c_{3}$ et $c_{2}$ n'est pas un arc étal. L'extrémité de $c_{2}$ dans $\left[s_{1} s_{3}\right]$ est encadrée par les extrémités de deux courbes singulières de $\xi Q_{1}$. À nouveau, on note $c_{1}$ celle dont l'extrémité est située après $\partial c_{2} \cap\left[s_{1} s_{3}\right]$ pour l'orientation de $\left[s_{1} s_{3}\right]$ induite par celle de la face $\left(s_{1} s_{3} s_{4}\right)$. Comme l'holonomie de toute courbe d'holonomie vaut -1 , l'extrémité $c_{1} \cap\left[s_{1} s_{2}\right]$ est voisine de celle de $c_{3}$, comme sur la figure 5 (ce qui ne serait pas le cas si on avait choisit $c_{1}, c_{2}$ et $c_{3}$ pour construire un arc étal). Soit $U_{1}$, $U_{2}$ et $U_{3}$ des petits voisinages tubulaires de $c_{1}, c_{2}$ et $c_{3}$ dans respectivement $Q_{1}, Q_{2}$ et $Q_{3}$. On note de plus $V_{1}, V_{2}$ et $V_{3}$ des voisinages, dans $\partial G$, des arcs délimités dans les arêtes $\left[s_{1} s_{3}\right]$, $\left[s_{1} s_{4}\right]$ et $\left[s_{1} s_{2}\right]$ par $\bigcup_{1 \leq i \leq 3}\left(\partial c_{i}\right)$. Les faces verticales recherchées sont obtenues par un lissage de

$$
U_{1} \cup U_{2} \cup U_{3} \cup V_{1} \cup V_{2} \cup V_{3}
$$

Elles sont par construction incluses dans $Q_{1} \cup Q_{2} \cup Q_{3}$ et elles ne rencontrent donc pas $P_{G} \cup \Lambda$. Ce sont les faces verticales d'un prisme fibré $P^{\prime}$ inclus dans $G$ qui ne rencontre pas $P_{G} \cup \Lambda$. Sur la figure 5, les faces verticales de $P^{\prime}$ sont délimitées par les traits pointillés ; les traits pleins représentent les lignes singulières du feuilletage des faces de $G$.

On déduit facilement de ce lemme que la famille $P$ n'était pas maximale : si $P^{\prime}$ n'est parallèle à aucun prisme de $P_{G}$, on ajoute $P^{\prime}$ à $P$; si $P^{\prime}$ est parallèle à un prisme $P_{1}$ de $P_{G}$, on remplace $P_{1}$ par un prisme qui contient $P^{\prime}$ et $P_{1}$ et dont le bord horizontal est contenu dans $\partial P^{\prime} \cup \partial P_{1}$.

Cas 1b. On peut construire un paquet $Q_{3} \subset\left(s_{1} s_{2} s_{4}\right) \backslash \Lambda$ entre $\left[s_{1} s_{4}\right] \cap Q_{2}$ et $\left[s_{2} s_{4}\right]$ d'épaisseur $N_{5}=N_{4}-10-C_{0}=f_{5}\left(N_{3}\right)$.

Dans ce cas, on considère le sous-paquet de $Q_{1}$ d'épaisseur $N_{4}-1$ contenant toutes les pièces issues de $Q_{2} \cap\left[s_{1} s_{3}\right]$. On rebaptise $Q_{1}$ ce nouveau paquet. On peut alors construire un paquet $Q_{0} \subset\left(s_{1} s_{2} s_{4}\right) \backslash \Lambda$ d'épaisseur au moins $N_{6}=N_{4}-1-10-C_{0}=f_{6}\left(N_{4}\right)$ entre 


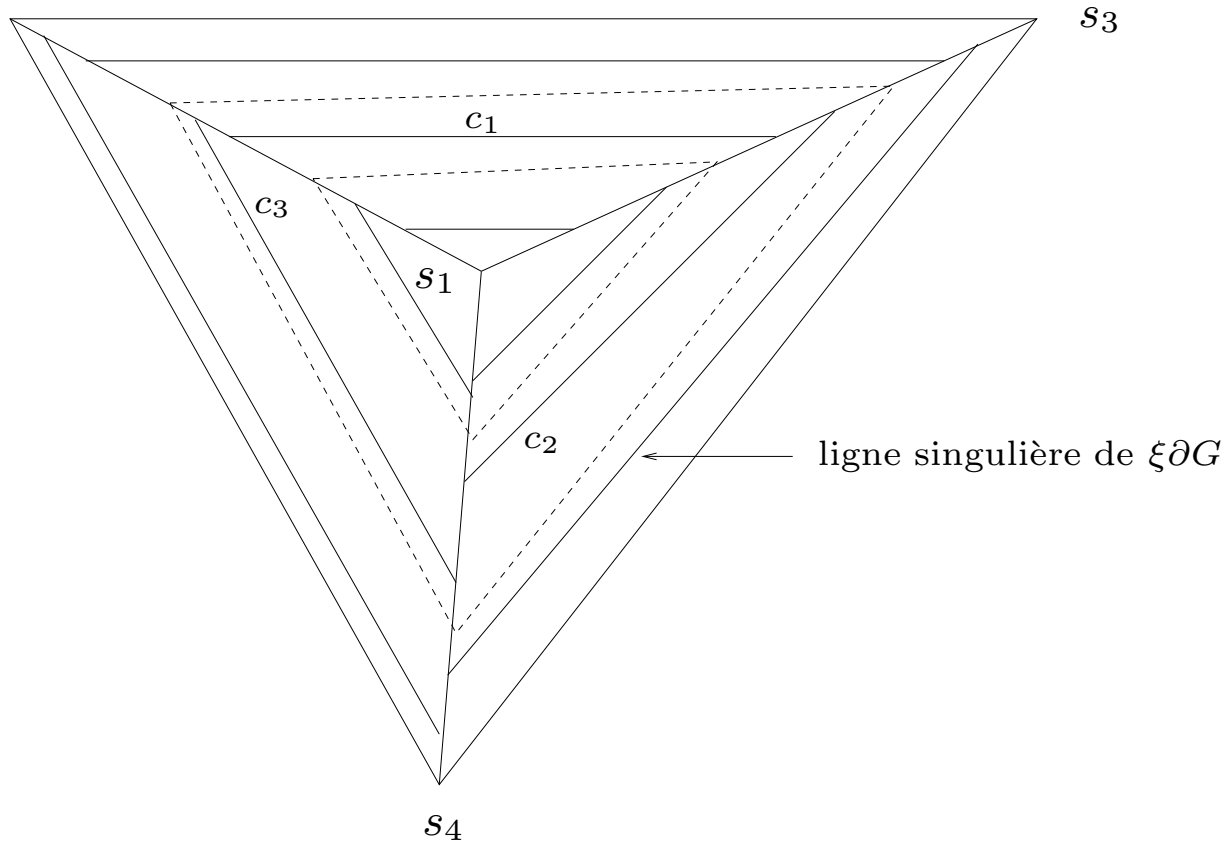

FIG. 5 - Les faces verticales de $P^{\prime}$.

$\left[s_{1} s_{2}\right] \cap Q_{1}$ et $\left[s_{2} s_{4}\right]$, sinon on se retrouve dans le cas $\left.1 a\right)$ avec un prisme fibré dont le bord vertical tourne autour de $s_{1}$.

On construit une courbe d'holonomie $\gamma=\gamma_{1} \cup \gamma_{2}$ pour laquelle $\gamma_{1}$ possède un voisinage de ses deux bouts dans la même face, ce qui donne une contradiction avec le lemme 2.17 au vu de la remarque 2.18. L'arc étal $\gamma_{1}$ est la concaténation de 4 arcs singuliers $c_{0} \subset Q_{0}$, $c_{1} \subset Q_{1}, c_{2} \subset Q_{2}, c_{3} \subset Q_{3}$, du feuilletage caractéristique des faces et de 3 portions d'arêtes qui relient leurs extrémités. L'arc $\gamma_{2}$ qui mesure l'holonomie sera pris dans [ $\left.s_{2} s_{4}\right]$. Pour cela, pour $N$ assez grand, on prend pour $c_{3}$ un arc singulier du feuilletage de $Q_{3}$ qui découpe $Q_{3}$ en deux paquets d'épaisseur au moins $20+C_{0}$ (pour être tranquille). Les choix des arcs singuliers $c_{0}, c_{1}$ et $c_{2}$ de même que celui des trois arêtes sont alors imposés par la condition d'être étal. Par la position de $c_{3}$, on est assuré que $c_{2} \subset Q_{2}$ et $c_{1} \subset Q_{1}$. De plus, $c_{1}$ découpe $Q_{1}$ en deux paquets d'épaisseur au moins $19+C_{0}$. Comme au plus $14+C_{0}$ composantes de $\Gamma_{\left(s_{1} s_{2} s_{4}\right)}(\xi)$ (estimation lâche) ayant leur extrémités dans $Q_{1}$ ne sont pas dans $Q_{0}$, on a bien $c_{0} \subset Q_{0}$. C'est l'arc $\gamma_{1}$ recherché.

Cas 2. On peut construire un paquet $Q_{2} \subset\left(s_{1} s_{3} s_{4}\right) \backslash \Lambda$ d'épaisseur $N_{4}^{\prime}=f_{4}^{\prime}\left(N_{3}\right)$ entre $\left[s_{1} s_{3}\right] \cap Q_{1}$ et $\left[s_{3} s_{4}\right]$.

On obtient ensuite un paquet $Q_{3} \subset\left(s_{2} s_{3} s_{4}\right) \backslash \Lambda$ d'épaisseur au moins $N_{5}^{\prime}=f_{5}^{\prime}\left(N_{4}^{\prime}\right)$ entre $\left[s_{3} s_{4}\right] \cap Q_{2}$ et $\left[s_{2} s_{3}\right]$, ce qui, pour $N$ assez grand, ramène au cas 1$)$, ou entre $\left[s_{1} s_{4}\right] \cap Q_{2}$ et $\left[s_{2} s_{4}\right]$. À nouveau, le seul cas non traité est celui où on peut construire un paquet $Q_{4} \subset\left(s_{1} s_{2} s_{4}\right) \backslash \Lambda$ d'épaisseur au moins 11 entre $\left[s_{2} s_{4}\right] \cap Q_{3}$ et $\left[s_{1} s_{2}\right]$ (voir figure [6). Dans ce dernier cas, on construit, comme dans le cas $1 a$, un prisme fibré dont le bord vertical est dans $Q_{1} \cup Q_{2} \cup Q_{3} \cup Q_{4}$. Comme précédemment, la famille $\left(P_{i}\right)_{i}$ n'était donc pas maximale.

\subsection{Construction des domaines fibrés}

On démontre à présent le théorème 2.1. 


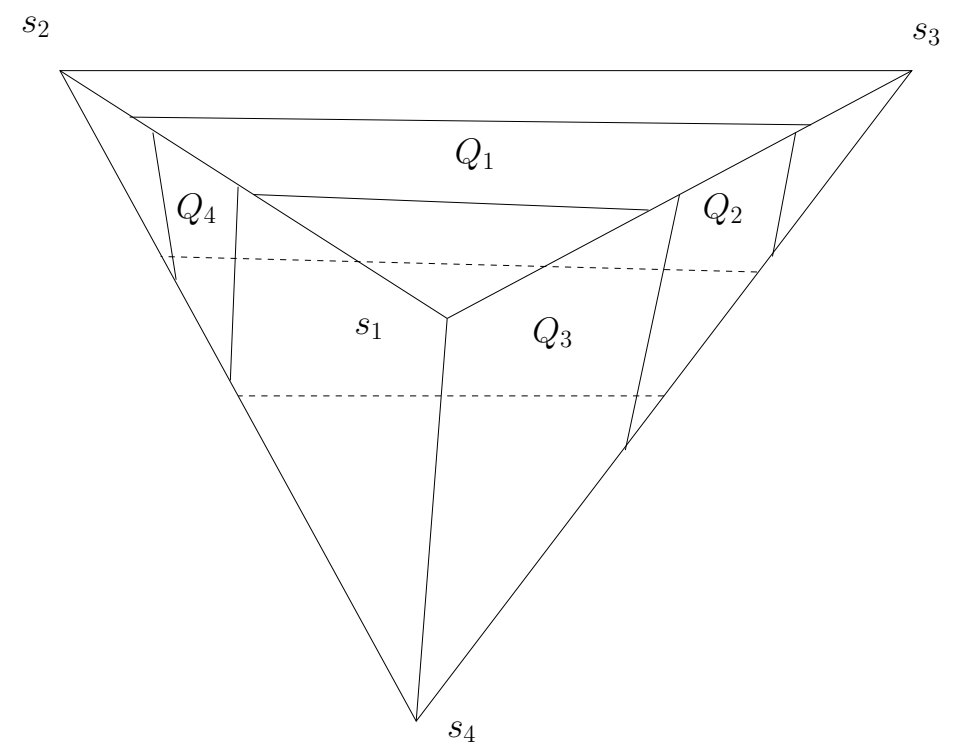

FIG. 6 - Le cas 2.

\subsubsection{Normalisation des faces verticales}

Les lemmes ci-dessous permettent, par des isotopies et des partitions successives de $\mathcal{X}$, d'affiner la normalisation des structures de $\mathcal{X}$. On rappelle que $\mathcal{X}$ est un ensemble complet de structures de contact tendues sur $V$ et que $\Delta$ est une triangulation de $V$, maniable et $\Lambda$-minimale pour tout $\xi \in \mathcal{X}$, toutes les structures de $\mathcal{X}$ étant égales le long de leur voisinage de sécurité commun $\Lambda$. En outre, les structures de $\mathcal{X}$ ont déjà subi une première normalisation et satisfont toutes aux conclusions du lemme 2.16.

Lemme 2.20. Il existe $C_{2}>0$ et un nombre fini de configurations de prismes fibrés $P^{1}, \ldots, P^{k}$ tels que, pour toute structure $\xi \in \mathcal{X}$, on puisse isotoper $\xi$, par une isotopie de $V$ stationnaire sur $\Lambda$ et préservant $\Delta$, en $\xi^{\prime}$ admettant un des $P^{j}$ pour configuration admissible et pour laquelle au plus $C_{2}$ composantes de $\Gamma_{\Delta^{2}}\left(\xi^{\prime}\right)$ ne soient pas incluses dans $\operatorname{Int}\left(\bigcup_{P \in P^{j}} P\right)$.

Une structure de contact $\xi \in \mathcal{X}$ qui vérifie ces propriétés pour une configuration $P^{j}$ sera dite portée par $P^{j}$.

Démonstration. À toute structure $\xi \in \mathcal{X}$, on peut associer un élément maximal $P(\xi)$ dans $\mathbf{P}_{\Delta, \xi}$. Le lemme 2.12 affirme qu'il n'y a qu'un nombre fini de classes d'isotopie de configurations de prismes fibrés. Une isotopie de $V$ fixant $\Delta$ et relative à $\Lambda$ permet d'envoyer chaque $P(\xi)$ sur un élément d'une sous-famille finie $P^{1}, \ldots, P^{k}$ de la famille $P(\xi)_{\xi \in \mathcal{X}}$. Cette isotopie transporte $\xi$ sur une structure $\xi^{\prime}$. Le lemme 2.14affirme l'existence de la borne universelle $C_{2}=C_{1}>O$.

Dans la suite, on remplace $\xi$ par $\xi^{\prime}$ comme représentant de la classe d'isotopie de $\xi$ dans $\mathcal{X}$. On démontre le théorème 2.1 pour l'ensemble $\mathcal{X}_{1}$ des structures $\xi \in \mathcal{X}$ portées par $P^{j}=P=\left(P_{i}\right)_{1 \leq i \leq n}$.

Lemme 2.21. Quitte à déformer chaque structure $\xi \in \mathcal{X}_{1}$ par une isotopie relative à $\Lambda$ qui préserve $\Delta$, il existe une partition de $\mathcal{X}_{1}$ en un nombre fini de sous ensembles $\mathcal{X}_{1}^{1}, \ldots, \mathcal{X}_{1}^{k}$ tels que, pour $j=1, \ldots, k$ : 
1. toutes les structures de $\mathcal{X}_{1}^{j}$ possèdent une même courbe de découpage sur les faces de $\Delta^{2} \backslash \operatorname{Int}\left(\bigcup_{1 \leq i \leq n} P_{i}\right) ;$

2. toutes les structures $\xi \in \mathcal{X}_{1}^{j}$ sont tangentes aux fibres des faces des prismes de $P$;

3. pour toute arête a, toutes les structures $\xi \in \mathcal{X}_{1}^{k}$ sont égales sur un voisinage de $a \backslash \operatorname{Int}\left(\bigcup_{1 \leq i \leq n} P_{i}\right)$.

Démonstration. Pour toute structure $\xi \in \mathcal{X}_{1}$, le nombre de composantes du découpage $\Gamma_{\Delta^{2} \backslash \operatorname{Int}\left(\cup_{1 \leq i \leq n} P_{i}\right)}(\xi)$ est inférieur à $C_{2}$. Le nombre de classes d'isotopie de multi-arcs

$$
\left(\Gamma_{\Delta^{2} \backslash \operatorname{Int}\left(\bigcup_{1 \leq i \leq n} P_{i}\right)}(\xi)\right)_{\xi \in \mathcal{X}_{1}}
$$

dans $\Delta^{2} \backslash \operatorname{Int}\left(\bigcup_{1 \leq i \leq n} P_{i}\right)$ est donc fini. Quitte à déformer chaque structure $\xi \in \mathcal{X}_{1}$ par une isotopie relative à $\Lambda$ qui préserve $\Delta$ et à partitionner $\mathcal{X}_{1}$ en $X_{1}^{1}, \ldots, X_{1}^{k}$, pour $j=1, \ldots, k$, on peut supposer que toutes les structures de $\mathcal{X}_{1}^{j}$ possèdent une même courbe de découpage sur $\Delta^{2} \backslash \operatorname{Int}\left(\bigcup_{1 \leq i \leq n} P_{i}\right)$. Les propriétés 2 et 3 découlent du lemme 2.16 (pour obtenir dans 3 l'égalité à partir du lemme 2.16, et pas seulement une cö̈ncidence des feuilletages legendriens, il faut encore utiliser la contractibilité de Diff ${ }^{+}([0,1])$ comme il est expliqué en détail dans la preuve du lemme 2.26).

On note $\mathcal{X}_{2}$ l'un des $\mathcal{X}_{1}^{j}, j=1, \ldots, k$, et on fixe $\zeta \in \mathcal{X}_{2}$. On est ramené à démontrer le théorème 2.1 pour $\mathcal{X}_{2}$.

Lemme 2.22. Toute structure $\xi \in \mathcal{X}_{2}$ est isotope à une structure maniable pour $\Delta$ et $\Lambda$-minimale $\xi^{\prime}$ qui cö̈ncide avec $\zeta$ le long de $\Delta^{2} \backslash \operatorname{Int}\left(\bigcup_{1 \leq i \leq n} P_{i}\right)$ et tangente aux fibres le long des faces verticales des polyèdres de $P$.

On note $\mathcal{X}_{3}$ l'ensemble obtenu par déformation de $\mathcal{X}_{2}$. Le long des portions de faces verticales qui ne sont pas incluses dans $\operatorname{Int}\left(\bigcup_{1 \leq i \leq n} P_{i}\right)$, toutes les structures $\xi \in \mathcal{X}_{3}$ sont à la fois égales et tangentes aux fibres.

Démonstration. Soit $R$ une composante de $H_{F} \backslash \Gamma_{H_{F}}$ incluse dans $H_{F} \backslash \operatorname{Int}\left(\bigcup_{1 \leq i \leq n} P_{i}\right)$. Les structures $\xi$ et $\zeta$ sont égales sur un voisinage $K$ de $\partial R \cap \partial F$ dans $R$, lequel $K$ est en outre feuilleté par des arcs legendriens. On rétrécit $R$ en $R_{0}$ en poussant chaque arc de $\partial R \cap \partial F$ sur un arc legendrien qui lui est parallèle dans $K$. Par application du lemme de réalisation de feuilletages 1.4 à $R_{0}$, on trouve une isotopie à support dans un voisinage de $R_{0}$, dont le temps 1 envoie $\xi$ sur $\zeta$ le long de $R_{0}$. Le point important est que le support de cette isotopie ne rencontre ni $\Lambda$ ni les faces autres que $F$, si bien $\Delta$ demeure maniable et $\Lambda$-minimale pour l'image de $\xi$.

\subsubsection{Normalisation des faces horizontales}

Pour chaque prisme $P_{i}, 1 \leq i \leq n$, on fixe un feuilletage non singulier $\mathcal{F}_{i}$ sur ses faces horizontales $Y_{i} \times\{0,1\}$, égal au germe de feuilletage tracé par $\zeta$ au bord $\left(\partial Y_{i}\right) \times\{0,1\}$ et transversal à une direction fixée de $Y_{i}$ (on munit $Y_{i}$ d'une structure affine). Un tel feuilletage non singulier existe grâce au fait que l'holonomie vaut -1 et donc que l'indice du germe de feuilletage caractéristique $\zeta Y_{i} \times\{j\}$ le long de $\left(\partial Y_{i}\right) \times\{j\}, j=0,1$ vaut 0 . Il détermine un germe de structure $\eta_{0}$ le long des faces horizontales.

Grâce à la remarque 2.15, on se place dans la situation où, pour tout $\xi \in \mathcal{X}_{3}$, toute face verticale des prismes de la configuration $P$ contient au moins 20 pièces. 
Lemme 2.23. Il existe une rétraction compacte $K=\bigcup_{1 \leq i \leq n} P_{i} \backslash N\left(\partial\left(\bigcup_{1 \leq i \leq n} P_{i}\right)\right)$ de $\bigcup_{1 \leq i \leq n} P_{i}$, une structure de contact $\zeta_{0}$ sur $V \backslash \operatorname{Int}(K)$ et pour tout $\xi \in \mathcal{X}_{3}$ une isotopie de $\bar{\xi}$ en $\xi^{\prime}$ qui vérifie:

1. $\xi^{\prime}=\zeta_{0}$ sur $V \backslash \operatorname{Int}(K)$;

2. $\xi^{\prime}$ est tangente aux fibres verticales de chaque $P_{i}$.

Démonstration. On commence par démontrer le lemme suivant :

Lemme 2.24. Soit $f: Y \times[-2,2] \rightarrow(V, \xi)$ un plongement dans une variété de contact tendue. On suppose que:

- chaque arc $\{x\} \times[-2,2]$, pour $x$ dans un voisinage de $\partial Y$, est legendrien pour $f^{*} \xi$;

- sur chaque face verticale, les courbes de découpage vont d'une arête verticale à l'autre ;

- l'holonomie de toute courbe d'holonomie tournant autour du bord vertical vaut -1 ; les arêtes de $f((\partial Y) \times\{ \pm 1\})$ sont transversales à $\xi$;

- il y a au moins quatre courbes de singularités sur chaque face verticale aux altitudes supérieures à 1 et inférieures à -1 ;

- les arêtes horizontales de $Y \times[-2,2]$ sont transversales à $f^{*} \xi$.

Pour tout feuilletage non singulier non singulier $\mathcal{F}$ tracé sur les faces horizontales de $Y \times[-1,1]$ qui est tangent à $f^{*} \xi$ au bord et transversal à une direction donnée de $Y$ (muni de sa structure affine), il existe une isotopie de $\xi$ relative au bord de l'image de $f$ en une structure $\xi^{\prime}$, telle que $f^{*} \xi^{\prime}$ trace le feuilletage $\mathcal{F}$ sur $Y \times\{ \pm 1\}$ et que chaque arc $\{x\} \times[-1,1]$ soit legendrien.

Démonstration. On munit $Y \times[-2,2]$ de la structure $f^{*} \xi$. Grâce à la présence de quatre arcs singuliers sur chaque face en dessous et au-dessus des altitudes -1 et 1 , on construit sur $(\partial Y) \times[1,2]$ et $(\partial Y) \times[-2,-1]$ une courbe d'holonomie legendrienne $\gamma_{ \pm 1}$ par concaténation de portions d'arêtes verticales et d'arcs de singularités sur les faces. On obtient que $t b\left(\gamma_{ \pm 1}\right)=-1$ car l'holonomie vaut -1 . On prend alors un disque convexe (de bord lisse par morceaux) $D_{ \pm 1} \subset Y \times[-2,2]$ qui s'appuie sur $\gamma_{ \pm 1}$ transversalement au bord vertical de $Y \times[-2,2]$. La portion de bord vertical comprise entre $\gamma_{-1}$ et $\gamma_{1}$ complétée par $D_{-1}$ et $D_{1}$ forme une sphère $S$ plongée dans $Y \times[-2,2]$.

On va construire un modèle de cette sphère dans $\mathbb{R}^{3}=\{(x, y, t)\}$. Pour cela, on munit $\mathbb{R}^{3}$ de la structure $\eta$ d'équation $\cos t d x-\sin t d y=0$. On fixe un triangle (ou un quadrilatère) $Y^{\prime}$ dans le plan des $(x, y)$, et on considère le polyèdre $Q=\left\{(x, y) \in Y^{\prime}, t \in[0,2 k \pi]\right\}$.

Comme l'holonomie de $\xi$ autour de $(\partial Y) \times[-2,2]$ est -1 , il existe $k \in \mathbb{R}$ et $Y^{\prime}$ pour que le bord vertical de ce polyèdre $Q$, avec le germe de structure $\eta$, soit conjugué à $(\partial Y) \times[-2,2]$ avec le germe de structure $f^{*} \xi$ par un germe de difféomorphisme de contact fibré (qui préserve la direction verticale, celle de $Q$ est dirigée par $\partial_{t}$ )

$$
\phi:(\partial Y) \times[-2,2] \rightarrow\left(\partial Y^{\prime}\right) \times[0,2 k \pi] .
$$

Il existe alors deux disques $D$ et $D^{\prime}$ qui s'appuient respectivement sur les courbes $\phi((\partial Y) \times\{ \pm 1\})$ du bord vertical du polyèdre $Q$, transversaux à la direction verticale $\partial_{t}$ et tels que $\phi$ s'étende en un difféomorphisme $\Phi: Y \times[-1,1] \rightarrow Q^{\prime}$, où $Q^{\prime}$ est le polyèdre de $\mathbb{R}^{3}$, dont le bord est union de $D$ et $D^{\prime}$ avec la portion de bord vertical de $Q$ comprise entre les courbes $\phi((\partial Y) \times\{ \pm 1\})$, avec les propriétés suivantes :

- les fibres verticales de $Y \times[-1,1]$ sont envoyées par $\Phi$ sur les fibres verticales de $Q^{\prime}$ (dirigées par $\partial_{t}$ qui sont legendriennes); 
- $\Phi_{*} \mathcal{F}$ est le feuilletage $\zeta\left(D \cup D^{\prime}\right)$.

Pour cela, on étend d'abord $\phi$ en un plongement fibré $\psi: Y \times[-1,1] \rightarrow Q$. Il ne reste plus qu'à relever les feuilletages $\psi_{*} \mathcal{F}$ de manière legendrienne transversalement à $\partial_{t}$ pour obtenir $D$ et $D^{\prime}$, puis à composer $\psi$ au but par l'extémité d'une isotopie qui amène, en glissant le long des fibres, l'image par $\psi$ des faces horizontales sur $D$ et $D^{\prime}$. Le fait que $\mathcal{F}$ soit transversal à une même direction sur les faces supérieure et inférieure assure que $D$ et $D^{\prime}$ ne se rencontrent pas.

Les courbes $\phi\left(\gamma_{ \pm 1}\right)$ sont legendriennes et d'invariant de Thurston-Bennequin -1 . On leur fait border dans $\left(\mathbb{R}^{3}, \eta\right)$ deux disques convexes qui ne rencontrent pas $Q^{\prime}$. On note $S^{\prime}$ la sphère construite comme précédemment à partir de ces disques convexes et de la portion de bord vertical qu'ils délimitent dans $Q$. D'après le lemme de réalisation de feuilletages, on peut choisir ces deux disques de sorte que $\phi$ s'étende en un difféomorphisme $\phi_{1}$ de $\left(S,\left.f^{*} \xi\right|_{S}\right) \rightarrow\left(S^{\prime},\left.\eta\right|_{S^{\prime}}\right)$.

D'après le théorème 1.1 d'Eliashberg, $\phi_{1}$ s'étend alors en un difféomorphisme de contact $\phi_{2}$ entre les boules bordées par ces sphères.

Le plongement $\phi_{2}^{-1} \circ \Phi: Y \times[-1,1] \rightarrow Y \times[-2,2]$ envoie $\mathcal{F}$ sur le feuilletage caractéristique de l'image des faces horizontales, et les fibres sur des courbes legendriennes. Il vaut l'identité sur le bord vertical et préserve l'orientation : il est isotope à l'identité relativement au bord de $Y \times[-2,2]$ par la restriction d'une isotopie de $Y \times[-2,2]$.

On considère l'image de $f^{*} \xi$ par cette isotopie que l'on propage à l'aide de $f$ dans $V$. Cette nouvelle structure donne le résultat souhaité.

Pour pousuivre, on applique le lemme 2.24 à chaque structure $\xi \in \mathcal{X}_{3}$ et au germe de structure $\eta_{0}$ le long des faces horizontales : il existe une isotopie de $\xi$ relative à $\Delta$ qui envoie $\xi$ sur une structure $\xi^{\prime}$ tangente aux fibres de $P$ et égale au germe $\eta_{0}$ sur les faces horizontales. On remplace chaque structure $\xi \in \mathcal{X}_{3}$ par la structure, à nouveau notée $\xi$, obtenue après cette première isotopie et on pioche une structure $\zeta_{0}$ dans $\mathcal{X}_{3}$.

Le long d'une fibre $I$ de $P_{i}$, toute structure $\xi$ tangente aux fibres est repérée par une fonction angle $\theta_{I}: I \rightarrow \mathbb{R} / \mathbb{Z}$ dans le fibré normal aux fibres, dont la dérivée est strictement positive. Dans un petit voisinage $N\left(\partial\left(\bigcup_{1 \leq i \leq n} P_{i}\right)\right) \cap\left(\bigcup_{1 \leq i \leq n} P_{i}\right)$ de $\partial\left(\bigcup_{1 \leq i \leq n} P_{i}\right)$ dans $\bigcup_{1 \leq i \leq n} P_{i}$, on peut après isotopie (toujours par contractibilité de $\operatorname{Diff}^{+}(I)$ ) rendre toutes ces fonctions angle égales à celle de $\zeta_{0} \in \mathcal{X}_{3}$. On réalise une telle isotopie sur chaque $\xi \in \mathcal{X}_{3}$ pour obtenir $\xi^{\prime} \in \mathcal{X}_{3}^{\prime}$.

Les prismes $P_{i}$ de la famille $P$ découpent chaque simplexe $G$ de $\Delta$ en polyèdres, homéomorphes à la boule, au bord desquels toutes les structures $\xi^{\prime} \in \mathcal{X}_{3}^{\prime}$ coïncident. Comme les structures de $\mathcal{X}_{3}^{\prime}$ sont tendues, le théorème d'unicité 1.1] d'Eliashberg donne, sur chaque polyèdre, une isotopie stationnaire au bord entre une structure quelconque $\xi^{\prime} \in \mathcal{X}_{3}^{\prime}$ et $\zeta_{0}$.

Corollaire 2.25. Il existe un domaine fibré à bord $(M, \tau)$ avec $K \subset M \subset \bigcup_{1 \leq i \leq n} P_{i}$, dont la fibration en intervalles $\tau$ est la restriction de la fibration de $\bigcup_{1 \leq i \leq n} P_{i}$. En particulier, selon la terminologie de la sous-section suivante, toutes les structures de $\mathcal{X}_{3}$ sont ajustées $\grave{a}(M, \tau, \zeta)$.

Démonstration. On retire à $\bigcup_{1 \leq i \leq n} P_{i}$ un petit voisinage fibré $U, U \cap K=\emptyset$, des portions d'arêtes qui ne sont pas dans $\operatorname{Int}\left(\bigcup_{1 \leq i \leq n} P_{i}\right)$. On note $\tau^{\prime}$ la fibration en intervalles de $\bigcup_{1 \leq i \leq n} P_{i}$. L'espace quotient $\Sigma=\left(\bigcup_{1 \leq i \leq n} P_{i} \backslash U\right) / \tau^{\prime}$ est une surface branchée dont le lieu singulier est non générique. Un lissage du bord de $\bigcup_{1 \leq i \leq n} P_{i} \backslash U$ transversal à la fibration au-dessus des points réguliers de la projection et une petite modification de $\bigcup_{1 \leq i \leq n} P_{i} \backslash U$ 
correspondant à une perturbation générique de $\Sigma$ pour obtenir un lieu de branchement générique permet d'obtenir le domaine fibré recherché.

Pour démontrer le théorème 2.1, il reste une difficulté : le domaine fibré du corollaire 2.25 est « à bord ». Dans la suite, on indique comment on se ramène à des voisinages fibrés de surfaces branchées sans bord.

\subsubsection{Structures de contact ajustées à un domaine fibré}

Soit $(M, \tau)$ un domaine fibré, voisinage d'une surface branchée à bord, et $X=M / \tau$ la surface branchée quotient. On note $\pi: M \rightarrow X$ et $\zeta$ une structure de contact sur $V \backslash \operatorname{Int}(M)$. Une structure de contact est ajustée à $(M, \tau, \zeta)$ si elle est égale à $\zeta$ hors de $\operatorname{Int}(M)$ et tangente à $\tau$ dans $M$.

Lemme 2.26. Toute structure de contact $\xi$ ajustée à $(M, \tau, \zeta)$ est déterminée, à isotopie près parmi les structures ajustées, par la fonction

$$
\left.a_{\xi}: \partial_{h} M: \rightarrow\right] 0, \infty[
$$

qui est continue sur chaque secteur et qui associe à chaque point p l'angle de rotation total de $\xi$ le long de la feuille de $\tau$ partant de p. (On mesure cet angle avec une métrique auxiliaire et l'holonomie de $\tau$.)

Démonstration. Ce lemme est une conséquence de la contractibilité de l'espace Diff ${ }^{+}(I)$ des difféomorphimes de l'intervalle qui préservent l'orientation. Soit $\xi_{0}$ et $\xi_{1}$ deux structures de contact sur $D^{2} \times I$ muni des coordonnées $((x, y), t)$, égales le long de $D^{2} \times\{0,1\}$, tangentes aux fibres $\{p\} \times I$ et pour lesquelles $a_{\xi_{0}}=a_{\xi_{1}}$ sur $D^{2}$. Elles sont alors données par $\alpha_{0}=\cos f_{0}(x, y, t) d x-\sin f_{0}(x, y, t) d y$ et $\alpha_{1}=\cos f_{1}(x, y, t) d x-\sin f_{1}(x, y, t) d y$ avec, pour $i=0,1, \frac{\partial f_{i}}{\partial t}>0$ et $f_{0}(x, y, i)=f_{1}(x, y, i)$. Par la contractibilité de $\operatorname{Diff}^{+}(I)$, il existe une famille à un paramètre de fonctions $f_{s}: D^{2} \times I \rightarrow \mathbb{R}, s \in[0,1]$, qui vérifient $\frac{\partial f_{s}}{\partial t}>0$ et qui sont indépendantes de $s \in[0,1]$ sur $D^{2} \times\{0,1\}$. Le chemin de formes de contact $\alpha_{s}=\cos f_{s}(x, y, t) d x-\sin f_{s}(x, y, t) d y$ donne une isotopie relative à $D^{2} \times\{0,1\}$ entre $\xi_{0}$ et $\xi_{1}$ parmi les structures tangentes à $\{*\} \times I$.

Pour démontrer le lemme, on donne une version relative de la discussion précédente. Soit $\xi$ et $\xi^{\prime}$ deux structures ajustées à $(M, \tau, \zeta)$, qui ont les mêmes fonctions angles $a_{\xi}=a_{\xi^{\prime}}$. Si $B$ est un secteur de $X$, alors $\partial B$ est un polygone $\partial B=\delta_{1} \cup \delta_{2} \cup \ldots \cup \delta_{m}$, où les $\delta_{i}$ sont les côtés successifs de $B$ qui se rencontrent le long des points triples de $X$. Comme $(B \times I) \cap \partial_{v} M$ consiste en la réunion de produits $\delta_{i} \times\left[a_{i}, b_{i}\right]$ où $\left[a_{i}, b_{i}\right] \subset[0,1]$, les structures $\xi$ et $\xi^{\prime}$ sont égales sur $\delta_{i} \times\left[a_{i}, b_{i}\right]$. On déforme d'abord $\xi$ en $\xi^{\prime}$ le long de $\delta_{i} \times\left([0,1] \backslash\left[a_{i}, b_{i}\right]\right)$ en utilisant la contractibilité de $\operatorname{Diff}^{+}(I)$, puis on effectue l'isotopie entre $\xi$ et $\xi^{\prime}$ sur $B \times I$ relativement à $(\partial B) \times I$.

Soit $\xi_{0}$ une structure de contact ajustée à $(M, \tau, \zeta)$ fixée.

Proposition 2.27. Les classes d'isotopie des structures de contact $\xi$ ajustées à $(M, \tau, \zeta)$ sont en bijection avec les fonctions

$$
w_{\xi}: \pi_{0}(\operatorname{Reg}(X)) \rightarrow \mathbb{Z}
$$

dites fonctions poids, vérifiant la condition

$$
w_{\xi}(R)>-\frac{1}{2 \pi} \inf \left(a_{\xi_{0}}\right)
$$


et les relations d'adjacence

$$
w_{\xi}\left(R_{1}\right)+w_{\xi}\left(R_{2}\right)=w_{\xi}\left(R_{3}\right)
$$

pour toute paire de feuillets réguliers $R_{1}, R_{2}$ de $X$ qui se joignent pour donner $R_{3}$.

Démonstration. Pour toute structure de contact $\xi$ ajustée à $(M, \tau, \zeta)$, on définit

$$
w_{\xi}=\frac{1}{2 \pi}\left(a_{\xi}-a_{\xi_{0}}\right) \text {. }
$$

Le poids $w_{\xi}(p)$ est un entier qui varie continûment sur les composantes de $\operatorname{Reg}(X)$. Il est donc constant sur $\operatorname{Reg}(X)$. La relation d'adjacence est donnée en regardant une fibre située au-dessus d'un point $p$ du lieu singulier de $X$ où $R_{1}$ et $R_{2}$ se rejoignent pour donner $R_{3}$. La condition de contact donne que $a_{\xi}>0$, ce qui fournit l'inégalité $w_{\xi}(R)>-\frac{1}{2 \pi} \inf \left(a_{\xi_{0}}\right)$.

Réciproquement, si on dispose d'une telle fonction poids, la fonction $a_{\xi}=w_{\xi}+a_{\xi_{0}}$ détermine une structure de contact qui convient.

\subsubsection{Le lemme d'élagage}

Lemme 2.28. [Lemme d'élagage] Soit $(M, \tau, \zeta)$ un domaine fibré et $\mathcal{X}$ un ensemble de structures de contact ajustées à $(M, \tau, \zeta)$. On suppose qu'il existe un réel $C$ et un point $p \in \partial_{h} M$ tels que $a_{\xi}(p)<C$ pour tout $\xi \in \mathcal{X}$ et on note $X_{1}, \ldots, X_{k}$ les adhérences des strates régulieres de $X=M / \tau$ qui contiennent $\pi(p)$. On peut alors trouver des structures de contact $\zeta_{1}, \ldots, \zeta_{l}$ sur le complémentaire du domaine fibré

$$
\left(M^{\prime}, \tau^{\prime}\right)=\left(M \backslash \operatorname{Int}\left(\bigcup_{j=1}^{k} \pi^{-1}\left(X_{j}\right)\right),\left.\tau\right|_{M^{\prime}}\right)
$$

telles que toute structure $\xi \in \mathcal{X}$ soit isotope à une structure ajustée à l'un des $\left(M^{\prime}, \tau^{\prime}, \zeta_{i}\right)$.

Démonstration. La borne sur $a_{\xi}$ donne une borne sur tous les poids $w_{\xi}\left(X_{i}\right), 1 \leq i \leq k$. Ceux-ci peuvent donc prendre seulement un nombre fini de valeurs. On peut partitionner $\mathcal{X}$ en $\mathcal{X}_{1}, \ldots, \mathcal{X}_{l}$, de sorte que toutes les $\xi \in \mathcal{X}_{j}$ donnent le même poids. Si $\zeta_{i}$ est une structure de $\mathcal{X}_{i}$, on peut alors, comme dans le lemme 2.26, trouver une isotopie, parmi les structures ajustées à $(M, \tau, \zeta)$, entre toute structure $\xi \in \mathcal{X}_{i}$ et une structure $\xi^{\prime}$ égale à $\zeta_{i} \operatorname{sur} \pi^{-1}\left(\bigcup_{1 \leq j \leq k} X_{j}\right)$.

L'opération d'élagage diminue strictement le nombre de secteurs de $X$. En la répétant un nombre fini de fois, on obtient donc l'ensemble vide.

Corollaire 2.29. Si $\mathcal{X}$ est un ensemble de structures de structures de contact ajustées à $(M, \tau, \zeta)$, alors il existe $\left(M_{1}, \tau_{1}, \zeta_{1}\right), \ldots,\left(M_{l}, \tau_{l}, \zeta_{l}\right)$ obtenues par élagage de $(M, \tau, \zeta)$ telles que, pour $1 \leq i \leq l, X_{i}=M_{i} / \tau_{i}$ soit une surface branchée sans bord, et que toute structure $\xi \in \mathcal{X}$ soit isotope à une structure $\xi^{\prime}$ ajustée à l'un des $\left(M_{i}, \tau_{i}, \zeta_{i}\right)$.

Démonstration. Tout point $p \in \partial X$ vérifie les hypothèses du lemme d'élagage. Par ailleurs, un élagage réduit strictement le nombre de secteurs de $X$. Un nombre fini d'applications du lemme d'élagage 2.28 conduit au résultat.

Le corollaire 2.29 termine la démonstration du théorème 2.1 . 


\section{$3 \quad$ Finitude homotopique}

On démontre ici le théorème 0.7. Le théorème 0.1 en résulte car une modification de Lutz à coefficient entier le long d'un tore ne change pas la classe d'homotopie dans les champs de plans.

Soit $V$ une variété close. Vu le théorème 2.1, il suffit de démontrer le théorème 0.7pour un ensemble $\mathcal{S}$ de structures de contact tendues toutes ajustées à un même domaine fibré $(M, \tau, \zeta)$. Soit $X$ la surface branchée $M / \tau$ avec son lieu singulier $\Theta$ et ses strates régulières $X_{0}, \ldots, X_{d}$. Étant donné $\xi_{0} \in \mathcal{S}$, chaque structure $\xi \in \mathcal{S}$ est déterminée (à déformation près parmi les structures ajustées) par son poids $w_{\xi}=\left(w_{\xi}\left(X_{1}\right), \ldots, w_{\xi}\left(X_{d}\right)\right) \in \mathbb{Z}^{d}$ relatif à $\xi_{0}$. Par application du lemme d'élagage et quitte à effectuer une partition de $\mathcal{S}$, on se ramène au cas où, pour tout $\xi \in \mathcal{S}$, le poids $w_{\xi}$ appartient à $\mathbb{N}^{d}$. $\grave{A}$ chaque arête lisse $C$ de $\Theta$, on associe l'équation linéaire $x_{i}=x_{j}+x_{k}$ sur $\mathbb{R}^{d}$ où $X_{j}$ et $X_{k}$ sont les secteurs de $X$ qui se joignent le long de $C$ pour former $X_{i}$. Soit $W$ le sous-espace vectoriel de $\mathbb{R}^{d}$ des solutions de ce système. Chaque poids $w_{\xi}$ appartient à $W \cap \mathbb{N}^{d}$.

On note $\preceq$ l'ordre partiel défini sur $\mathbb{Z}^{d}$ par

$$
\left(x_{1}, \ldots, x_{d}\right) \preceq\left(y_{1}, \ldots, y_{d}\right) \quad \text { si } \quad x_{i} \leq y_{i} \text { pour } 1 \leq i \leq d .
$$

Lemme 3.1. Soit $W$ un sous-espace vectoriel de $\mathbb{R}^{d}$. Les éléments minimaux de $W \cap \mathbb{N}^{d}$ pour l'ordre partiel $\preceq$ sont en nombre fini et engendrent $W \cap \mathbb{N}^{d}$.

Démonstration. Pour montrer que les éléments minimaux de $W \cap \mathbb{N}^{d}$ sont en nombre fini, on raisonne par récurrence sur $d$. Lorsque $d=1$, on a un unique élément minimal. On suppose le résultat démontré pour tout sous-espace de $\mathbb{R}^{d-1}, d-1 \geq 1$. Soit maintenant $W$ un sous-espace de $\mathbb{R}^{d}, d \geq 2$. On raisonne par l'absurde en supposant que $W \cap \mathbb{N}^{d}$ possède une infinité d'éléments minimaux. Quitte a permuter l'ordre des coordonnées, on peut alors trouver une suite d'éléments minimaux $\left(v^{i}\right)_{i \in \mathbb{N}}, v^{i}=\left(v_{1}^{i}, \ldots, v_{d}^{i}\right)$, de $W \cap \mathbb{N}^{d}$ pour laquelle la suite des dernières coordonnées $\left(v_{d}^{i}\right)_{i \in \mathbb{N}}$ tend vers l'infini. On note $\pi: \mathbb{R}^{d} \rightarrow \mathbb{R}^{d-1}$ la projection sur les $d-1$ premières coordonnées. En appliquant l'hypothèse de récurrence à $\pi(W)$, on obtient un nombre fini d'éléments $u_{1}, \ldots, u_{n}$ de $\mathbb{N}^{d}$ dont les images par $\pi$ sont les éléments minimaux de $\pi(W) \cap \mathbb{N}^{d-1}$. Si $i \in \mathbb{N}$ est assez grand, $v_{d}^{i}$ est supérieur à chacune des dernières coordonnées des vecteurs $u_{1}, \ldots, u_{n}$ et en particulier, $v^{i}$ est supérieur à un des $u_{j}$. C'est une contradiction.

Si $u \in W \cap \mathbb{N}^{d}$ n'est pas un élément minimal, il est supérieur à l'un d'entre eux $u_{i}$. Soit le vecteur $u-u_{i}$ est minimal, soit on peut à nouveau lui retirer un des $u_{j}, j=1, \ldots n$. En un nombre fini d'étape, on écrit $u$ comme une somme d'éléments minimaux.

On note $u_{1}, \ldots, u_{k}$ les éléments minimaux de $W \cap \mathbb{N}^{d}$ donnés par le lemme 3.1. Par ailleurs,

Lemme 3.2. Les classes d'isotopie des surfaces compactes plongées dans $M$ transversalement à $\tau$ sont en bijection avec les éléments non nuls de $W \cap \mathbb{N}^{d}$.

Si $\mathcal{T}$ est une surface portée par $(M, \tau)$, on note $w_{\mathcal{T}}=\left(w_{\mathcal{T}}\left(X_{1}\right), \ldots, w_{\mathcal{T}}\left(X_{d}\right)\right)$ son poids, où $w_{\mathcal{T}}\left(X_{i}\right)$ est le nombre de composantes de $\mathcal{T} \cap \pi^{-1}\left(X_{i}\right)$ et $\pi$ la projection $M \rightarrow X=M / \tau$.

Remarque 3.3. Si $X$ est compact sans bord et $\mathcal{S}$ non vide, les surfaces compactes portées par $X$ - c'est-à-dire plongées dans $M$ transversalement à $\tau$ - sont closes et transversales aux structures de contact de $\mathcal{S}$. Leurs composantes connexes sont donc des tores et/ou des bouteilles de Klein. 
Soit $T_{1}, \ldots, T_{k}$ les surfaces portées par $(M, \tau)$ et correspondant aux éléments $u_{1}, \ldots, u_{k}$ de $W \cap \mathbb{N}^{d}$. On suppose que $T_{1}, \ldots, T_{l}$ sont des tores et que $T_{l+1}, \ldots, T_{k}$ sont des bouteilles de Klein. Lorsque $T_{i}$ est une bouteille de Klein, on note $T_{i}^{\prime}$ le tore, toujours porté par $(M, \tau)$, bordant un de ses petits voisinages tubulaires $N\left(T_{i}\right)$. Le poids $u_{i}^{\prime}$ de $T_{i}^{\prime}$ vaut $2 u_{i}$.

Lemme 3.4. Toute structure de contact $\xi \in \mathcal{S}$ est obtenue à partir de $\xi_{0}$ par modification de Lutz sur les tores $T_{i}, 1 \leq i \leq l$ et $T_{i}^{\prime}, l+1 \leq i \leq k$.

Démonstration. Soit $\xi \in \mathcal{S}$. On a $w_{\xi}=\sum_{i=1}^{k} n_{i}(\xi) u_{i}$, avec $n_{i}(\xi) \in \mathbb{N}$. La structure obtenue à partir de $\xi_{0}$ par chirurgie de Lutz de coefficient $n_{i}(\xi)$ sur $T_{i}$ lorsque $T_{i}$ est un tore et de coefficient $\frac{1}{2} n_{i}(\xi)$ sur $T_{i}^{\prime}=\partial N\left(T_{i}\right)$ lorsque $T_{i}$ est une bouteille de Klein est ajustée à $(M, \tau, \zeta)$ et a le même poids que $\xi$. Elle lui est donc isotope relativement à $\partial M$.

Même si les coefficients de chirurgie apparaissant dans le lemme 3.4 sont des demientiers, celui-ci implique le théorème 0.7 : on ajoute comme «structures de base » à $\xi_{0}$

les $2^{k-l-1}$ structures obtenues en faisant ou non des modifications de Lutz de coefficient $\frac{1}{2}$ le long des tores $T_{i}^{\prime}, l+1 \leq i \leq k$. Toutes les structures de $\mathcal{S}$ sont alors obtenues à partir de l'une d'entre elles par modifications de Lutz à coefficients entiers sur les collections de tores $\left(T_{i}\right)_{1 \leq i \leq l}$ et $\left(T_{i}^{\prime}\right)_{l+1 \leq i \leq k}$.

\section{Finitude géométrique}

On démontre maintenant le théorème 0.6. Comme précédemment, grâce au théorème 2.1 il suffit d'établir le résultat pour un ensemble $\mathcal{S}$ de structures tendues toutes ajustées à un même moule $(M, \tau, \zeta)$. On fixe une structure de référence $\xi_{0} \in \mathcal{S}$, toute autre $\xi \in \mathcal{S}$ étant alors déterminée par son poids $w_{\xi}$ dont on peut supposer, sauf pour $\xi_{0}$, qu'il est dans $(\mathbb{N} \backslash\{0\})^{d}$ (voir le lemme d'élagage 2.28). Pour une telle structure $\xi \in \mathcal{S} \backslash\left\{\xi_{0}\right\}$, le poids $w_{\xi}$ correspond à une surface close $T$ pleinement portée par $(M, \tau)$. Chaque composante de $T$ est un tore ou une bouteille de Klein.

Lemme 4.1. Il existe une surface orientée $\mathcal{T}$ pleinement portée par $(M, \tau)$ qui contient le bord horizontal de $M$ comme sous-surface orientée.

Démonstration. Le domaine $M$ contient une surface $T$ pleinement portée, par exemple celle que détermine le poids d'une structure $\xi \in \mathcal{S}$. En doublant $T$ si nécéssaire et en remplaçant chaque bouteille de Klein par le bord d'un de ses voisinages tubulaires, on s'assure que les composantes de $T$ sont des tores et que $T$ intersecte au moins deux fois chaque fibre de $M$. Parmi ces intersections, deux sont les plus proches de $\partial_{h} M$. On pousse les composantes connexes de $T$ contenant ces intersections extriêmes jusque dans $\partial M$. Quitte à doubler $T$ à nouveau pour pouvoir l'orienter comme voulu, on obtient la surface cherchée.

\subsection{Le lemme du degré}

Soit $A$ une composante connexe de $\partial_{v} M$ et $C$ une composante du bord de $A$. On définit le degré $\operatorname{deg}(A)$ de $A$ comme la valeur absolue du degré de l'image de $\zeta_{x}$ par rapport à $T_{x} A$ dans le quotient $T_{x} V / T_{x} \tau$ lorsque $x$ parcourt $C$. 
Assertion 4.2. Quitte à modifier $(M, \tau, \zeta)$ par élagage et isotopie, on peut supposer que pour chaque composante $A$ de $\partial_{v} M$ et chaque composante $C$ de $\partial A$, il y a exactement $2 \operatorname{deg}(A)$ points le long de $C$ où $T_{x} A$ cö̈ncide avec $\zeta_{x}$. En particulier, si $\operatorname{deg}(A)=0$, le feuilletage $\zeta A$ contient une courbe de singularités isotope à l'âme de A.

Démonstration. On étend $A$ le long des fibres legendriennes de $M$ jusqu'à obtenir un anneau $A^{\prime}$, où $A^{\prime} \backslash A \subset M$, pour lequel les structures $\left.\xi\right|_{A^{\prime}}$ sont égales pour tout $\xi \in \mathcal{S}$ et la condition de l'assertion est vérifiée le long de $A^{\prime}$. Cette extension est rendue possible par le fait qu'on peut supposer que toutes les structures $\xi \in \mathcal{S}$ ont une fonction poids minorée par une constante $c>0$ que l'on peut choisir arbitrairement grande (quitte à procéder à des partitions de $\mathcal{S}$ et à élaguer $M)$. On considère alors un épaississement $A^{\prime} \times[0,1]$ de $A^{\prime}$ dans $M$, où $A^{\prime} \times\{1\}=A^{\prime}$ et où chaque $\xi \in \mathcal{S}$ est $I$-invariante (en particulier chaque $A^{\prime} \times\{t\}$ est feuilleté par des intervalles legendriens). On prend pour nouveau domaine fibré $M^{\prime}=M \backslash\left(A^{\prime} \times I\right)$ dont on lisse les coins de sorte que $A^{\prime} \times\{0\} \subset M^{\prime}$ soit une composante du bord vertical de $M^{\prime}$.

Dorénavant, toutes les composantes de $\partial_{v} M$ sont réputées satisfaire aux conclusions de l'assertion 4.2 .

Lemme 4.3. [Lemme du degré] Il existe des domaines fibrés $\left(M_{i}, \tau_{i}, \zeta_{i}\right)_{1 \leq i \leq k}$ obtenus par élagage de $(M, \tau, \zeta)$ avec les propriétés suivantes:

- toute structure de contact $\xi \in \mathcal{S}$ est conjuguée, par un produit de twists de Dehn et d'isotopies, à une structure ajustée à l'un des $\left(M_{i}, \tau_{i}, \zeta_{i}\right)$;

- tout domaine fibré $\left(M_{i}, \tau_{i}\right)$ porte pleinement une surface $\mathcal{T}_{i}$ comme dans le lemme 4.1];

- toutes les composantes de $\partial_{v} M_{i}$ satisfont aux conclusions de l'assertion 4.2

- toute composante de $\partial_{v} M_{i}$ de degré non nul intersecte $\mathcal{T}_{i}$ le long de courbes contractibles dans $\mathcal{T}_{i}$.

Démonstration. Soit $T$ une composante de $\mathcal{T}$ qui intersecte une composante $A$ de $\partial_{v} M$ avec $\operatorname{deg}(A) \neq 0$ le long de $c$. On suppose que $c$ est non contractible sur $T$. Pour tout $\xi \in \mathcal{S}$ avec $w_{\xi}$ suffisamment grande, il existe un plongement $\phi: T \times[0,1] \rightarrow M$, où $\phi(T, 0)=T$ et $\phi^{*} \xi$ est donnée par $\cos (f(x, y)+2 \pi t) d x-\sin (f(x, y)+2 \pi t) d y=0$. Ici, les coordonnées sur $T \times[0,1]=\mathbb{R}^{2} / \mathbb{Z}^{2} \times[0,1]$ sont $(x, y, t)$, et $f$ est une fonction à valeurs dans le cercle $T \rightarrow \mathbb{R} / 2 \pi \mathbb{Z}$. On a alors la propriété suivante :

Assertion 4.4. Il existe un tore $T^{\prime} \subset \phi(T \times[0,1])$ isotope à $T$ et transversal aux fibres legendriennes tel que $T^{\prime}$ soit convexe et $\# \Gamma_{T^{\prime}}(\xi) \leq 2 \operatorname{deg}(A)$.

Démonstration de l'assertion 4.4. Après une perturbation $\mathcal{C}^{\infty}$-petite de $T$, on peut supposer que $T$ est convexe. Comme $T \pitchfork \xi$ pour tout $\xi \in \mathcal{S}$, le feuilletage caractéristique $\xi T$ est non singulier, et donc $\# \Gamma_{T}(\xi)$ est égal au nombre d'orbites fermées $\gamma_{i}$ de $\xi T$. Les $T \backslash \bigcup_{i} \gamma_{i}$ sont des composantes annulaires qui sont soit de Reeb (ne possèdent pas d'arc transversal s'appuyant au bord et qui intersecte toutes les feuilles) ou tendues (il existe un tel arc transversal). On peut supposer que $c$ est transversal à $\bigcup_{i} \gamma_{i}$. En analysant les composantes de $c \backslash \bigcup_{i} \gamma_{i}$, tout arc (séparant ou non séparant) situé dans une composante de Reeb donne au moins une tangence, tandis que les arcs situés dans une composante tendue ne contribuent pas nécéssairement. Donc le nombre de composantes de Reeb est majoré par $2 \operatorname{deg}(A)$ (= le nombre de tangences de $c$ ), si les orbites $\gamma_{i}$ ont une intersection non triviale avec $c$. Pour voir que les orbites $\gamma_{i}$ ont une intersection géométrique non triviale avec $c$, on observe que $2 \operatorname{deg}(A)$, qui est le décompte algébrique du nombre de 
tangences entre $c$ et $\xi T$, est invariant par isotopie. Si le nombre d'intersection géométrique est nul, alors le degré doit aussi être nul. Pour conclure, toutes les composantes tendues peuvent être supprimées en isotopant $T$ à une distance bornée dans $\phi(T \times[0,1])$. On peut également remarquer que l'assertion 4.2 implique que les composantes de Reeb pointent dans la même direction.

Le point clé pour le tore convexe $T$ modifié comme dans le lemme précédent est que $\# \Gamma_{T}(\xi)$ est borné indépendemment du choix de $\xi \in \mathcal{S}$. On suppose que $\xi \in \mathcal{S}$ satisfait $w_{\xi} \gg n w_{T}$, où $n=\frac{1}{2} \# \Gamma_{T}(\xi)$. Alors il existe un plongement $\psi: T \times[0, n] \rightarrow N(\mathcal{B})$, où $T \times\{0\}=T^{\prime}$ et $\psi^{*} \xi$ est donnée $\operatorname{par} \cos (g(x, y)+2 \pi t) d x-\sin (g(x, y)+2 \pi t) d y=0$. Si on excise $\psi(T \times[0, n])$ et on recolle $\psi(T \times\{0\})$ avec $\psi(T \times\{n\})$ via l'identification naturelle donnée par la fibration legendrienne, on obtient une structure de contact $\xi^{\prime}$ correspondant au poids $w_{\xi}-n w_{T}$. Maintenant, $\xi$ et $\xi^{\prime}$ sont isomorphes car elles diffèrent par des twists de Dehn le long du tore $T$. Pour cette raison, on peut réduire inductivement $w_{\xi} \rightarrow w_{\xi}-n w_{T}$ jusqu'à ce qu'un secteur de $X$ ait un petit poids. Un tel secteur peut alors être élagué.

En appliquant le lemme du degré à $(M, \tau, \zeta)$, on se ramène au cas où $(M, \tau, \zeta)$ est l'un des $\left(M_{i}, \tau_{i}, \zeta_{i}\right)$. On peut encore améliorer $M$ par élagage grâce à la proposition suivante :

Proposition 4.5. Après des élagages successifs, on peut supposer, quitte à conjuguer les structures de $\mathcal{S}$ par des produits de twists de Dehn, que, pour toute composante $A$ de $\partial_{v} M$

1. $\operatorname{deg}(A)=0$ si et seulement si les deux composantes de $\partial A$ sont non contractibles dans $\mathcal{T}$.

2. $\operatorname{deg}(A)=1$ si et seulement si les deux composantes de $\partial A$ bordent des disques dans $\mathcal{T}$.

Démonstration. Par le lemme du degré, si une composante de $\partial A$ est non contractible, alors $\operatorname{deg}(A)=0$. À l'inverse, si une composante $c$ de $\partial A$ borde un disque $D$ dans $\mathcal{T}$, alors par l'assertion 4.2 et la non singularité du feuilletage caractéristique de $D$, il ne peut y avoir que deux points le long de $c$ pour lesquels $T_{x} A=\zeta_{x}$. Donc $\operatorname{deg}(A)=1$. Ainsi, soit les deux composantes de $\partial A$ sont non contractibles dans $\mathcal{T}$, soit elles bordent toutes deux un disque.

Remarque 4.6. Si les deux composantes de $\partial A$ bordent un disque, alors ces disques doivent être du même côté de $A$, sinon la sphère constituée de l'union de ces deux disques avec $A$ pourrait être lissée en une sphère transversale à $\xi_{0} \in \mathcal{S}$.

\section{2 Élimination des disques de contact}

Dans cette partie, on simplifie le domaine fibré $M$ en éliminant les disques de contact. Un disque de contact est un disque proprement plongé $D \subset M$, transversal aux fibres et dont le bord est dans $\partial_{v} M$.

Lemme 4.7. Soit $A$ une composante de $\partial_{v} M$. S'il existe un disque de contact $D$ dont le bord est dans $A$, alors les composantes $c_{1}, c_{2}$ de $\partial A$ bordent des disques $D_{1}, D_{2} \subset \mathcal{T}$ de sorte que $D_{i}$ soit dans l'intérieur de $M$ près de $\partial D_{i}$. 
Démonstration. Comme $A$ admet un disque de contact $D$ et que le feuilletage caractéristique de $D$ est non singulier, $\operatorname{deg}(A)$ doit être égal à un. Par le lemme du degré, $c_{i}$ doit border un disque $D_{i}$ dans $\mathcal{T}$. On note que $D_{i}$ ne peut pas être dans la « direction opposée » à $D$, c'est-à-dire que $D_{i}$ ne peut pas contenir la composante de $\partial_{h} M$ adjacente à $c_{i}$. Dans le cas contraire, $D \cup D_{i}$ (augmenté de parties de $A$ et après lissage) formerait une sphère immergée transversale à la fibration legendrienne, ce qui est impossible.

Remarque 4.8. Il est possible que $D_{1} \subset D_{2}$ où vice-versa.

Proposition 4.9. Soit $V$ une variété close et irréductible de dimension trois. Il existe un nombre fini de paires $\left(N_{i}, \zeta_{i}\right), i=1, \ldots, k$, satisfaisant aux conditions suivantes :

1. $N_{i} \subset V$ est une union finie de tores épaissis $T^{2} \times[0,1]$, d'anneaux épaissis $A \times[0,1]$ et de voisinages de bouteilles de Klein $N(K)$, où chaque $A \times\{j\}, j=0,1$, est collé de façon incompressible sur un $\partial\left(T^{2} \times[0,1]\right)$ ou sur un $\partial N(K)$, et où certaines composantes de bord de $T^{2} \times I$ peuvent être identifiées entre elles ou avec un $\partial N(K)$;

2. $\zeta_{i}$ est une structure de contact tendue sur $V \backslash \operatorname{Int}\left(N_{i}\right)$;

3. toute structure de contact tendue sur $V$ est conjuguée, par un produit de twists de Dehn et d'isotopies à une structure égale à l'une des $\zeta_{i}$ sur $V \backslash \operatorname{Int}\left(N_{i}\right)$.

Démonstration. On élimine d'abord les disques de contact de $M$, tout en préservant la condition que $M$ porte pleinement une union de tores $\mathcal{T}$. (Voir la remarque 4.10 cidessous.) S'il y a un disque de contact pour $A$, alors en utilisant le lemme 4.7, on peut le remplacer par des disques de contact (pour $A$ ) $D_{1}$ et $D_{2}$ dans $\mathcal{T}$. Sans perte de généralité, on suppose que $D_{1}$ est un disque de contact le plus intérieur pour $\mathcal{T}$. Alors, soit $D_{1}$ et $D_{2}$ sont disjoints, soit $D_{1} \subset D_{2}$. Comme $D_{1}$ peut contenir des disques de $\partial_{h} M$, on le pousse légèrement le long des fibres pour l'éloigner de $\partial_{h} M \cap \operatorname{Int}\left(D_{1}\right)$. On appelle $D_{1}^{\prime}$ cette déformation de $D_{1}$. On change alors $M$ en $M \backslash D_{1}^{\prime}, \mathcal{T}$ en $\left(\mathcal{T} \backslash D_{1}\right) \cup D_{1}^{\prime}$, et $D_{2}$ en $\left(D_{2} \backslash D_{1}\right) \cup D_{1}^{\prime}$ si $D_{1} \subset D_{2}$ (puis, après le paragraphe ci-dessous on les rebaptise $M, \mathcal{T}$ et $\left.D_{2}\right)$.

On explique maintenant comment transformer $D_{1}^{\prime}$ en une surface convexe (de bord legendrien), de sorte que la structure de contact $\zeta$ s'étende de manière unique en une structure de contact tendue sur $M \cup N\left(D_{1}^{\prime}\right)$, c'est-à-dire de sorte qu'on puisse isotoper toutes les structures $\xi \in \mathcal{S}$ sur $M$ relativement à $\partial M$ pour les faire coïncider sur (un voisinage de) $D_{1}^{\prime}$. Après un lissage des coins de $\partial M$ et une perturbation générique, $\partial M$ devient convexe. Le fait que $\operatorname{deg}(A)=1$ se traduit par le fait qu'on peut trouver une isotopie de $\zeta$ près de $\partial M$ (et donc une isotopie concomitante des structures $\xi \in \mathcal{S}$ ) qui rende $\partial D_{1}^{\prime}$ legendrien avec $t b\left(\partial D_{1}^{\prime}\right)=-1$. Dans ce cas, si $D_{1}^{\prime}$ est perturbé en une surface convexe de bord legendrien, il n'y a qu'une seule possibilité pour $\Gamma_{D_{1}^{\prime}}(\xi)$ à isotopie près. Dès lors, en appliquant le lemme de réalisation de feuilletages on peut supposer que toutes les structures $\xi \in \mathcal{S}$ donnent le même germe le long de $D_{1}^{\prime}$.

Comme (le nouveau) $\mathcal{T}$ n'est pas pleinement porté par le (nouveau) $M$ (voir la remarque 4.10 : la fibration persiste topologiquement, de même que la notion de surface portée), on modifie $\mathcal{T}$ de la façon suivante : soit $T$ la composante de $\mathcal{T}$ qui contient $D_{2}$, et soit $T^{\prime}$ une déformation parallèle de $T$. On remplace $T$ par un lissage de $\left(T \backslash D_{2}\right) \cup A \cup D_{1}^{\prime}$. Si on dédouble ce nouveau tore, on obtient une nouvelle collection de surfaces $\mathcal{T}$ qui contient $\partial_{h} M$. Comme $\partial_{v} M$ est constitué d'un nombre fini de composantes, on élimine ainsi tous les disques de contact en un nombre fini d'opérations. On observe que les composantes de $\partial_{v} M \cap \mathcal{T}$ qui étaient non homotope à zéro (resp. homotope) dans $\mathcal{T}$ le demeurent. 
Une fois éliminés tous les disques de contact, on étudie les composantes de $\partial_{h} M$. Elles sont de trois types: (i) disques, (ii) anneaux incompressibles dans $\mathcal{T}$ et (iii) tores. Tous les disques de $\partial_{h} M$ peuvent être éliminés de la façon suivante. Soit $D$ une composante de $\partial_{h} M$ difféomorphe à un disque et $A$ un anneau qui partage une composante de bord avec $D$. Le degré $\operatorname{deg}(A)$ doit être non nul et si $S$ est une composante de $\partial_{h} M$ qui intersecte l'autre composante de bord de $A$, alors, par le lemme du degré, $S$ ne peut pas être un anneau incompressible dans $\mathcal{T}$. Donc $S$ est aussi un disque. Mais alors $D \cup A \cup S$ est une sphère qui borde une boule $B^{3}$ d'un côté ou de l'autre. Dans un cas, on remplace $M$ par $M \cup B^{3}$ et dans l'autre par $M \backslash B^{3}$ (où on est sûr qu'à isotopie près toutes les structures de $\mathcal{S}$ coïncident d'après le théorème de classification 1.1 d'Eliashberg). À la fin du processus, tous les disques du bord horizontal sont éliminés. Ceci implique que toutes les composantes de $M \backslash \mathcal{T}$ sont des tores épaissis, des voisinages de bouteilles de Klein ou des anneaux épaissis qui sont collés de façon incompressible les uns aux autres.

Remarque 4.10. En éliminant les disques de contact, on perd le contrôle sur la fibration legendrienne, bien qu'elle persiste topologiquement. Ainsi, au lieu de considérer les classes de conjugaison de structures de contact tendues ajustées à un triplet $(M, \tau, \zeta)$, on doit considérer les classes de conjugaison de structures de contact sur $V$ qui cö̈ncident avec $\zeta$ sur $V \backslash \operatorname{Int}(N)$. Du fait de cette perte d'information, on doit s'appuyer sur un résultat de finitude pour une classe simple de variétés $N$, précisément lorsque $N$ est un fibré en cercles au-dessus d'une surface.

\subsection{Réduction aux fibrés en cercles}

Soit $(N, \zeta)=\left(N_{i}, \zeta_{i}\right)$ une paire comme dans la proposition 4.9 et $\mathcal{T}(N, \zeta)$ un ensemble de structures de contact tendues sur $V$ égales à $\zeta$ sur $V \backslash \operatorname{Int}(N)$ et de torsions bornées par $n$. L'objectif de cette partie est de démontrer la proposition suivante.

Proposition 4.11. Il existe une famille finie de paires $\left(N_{1}, \zeta_{1}\right), \ldots,\left(N_{l}, \zeta_{l}\right)$ satisfaisant aux conditions suivantes :

- les $N_{i}, i=1, \ldots, l$, sont des fibrés en cercles au-dessus de surfaces compactes à bord, éventuellement non orientables, dans $V$;

- $\zeta_{i}, i=1, \ldots, l$, est une structure de contact tendue sur $V \backslash \operatorname{Int}\left(N_{i}\right)$;

- toutes les composantes de $\Gamma_{\partial N_{i}}$ sont isotopes aux fibres de $N_{i}$;

- toute structure de $\mathcal{T}(N, \zeta)$ est conjuguée, par un produit de twists de Dehn et d'isotopies, à une structure égale à $\zeta_{i}$ sur $V \backslash \operatorname{Int}\left(N_{i}\right)$ pour un certain $i \in[1, l]$.

On observe déjà que la sous-variété $N$ n'est pas quelconque.

Lemme 4.12. La variété $N$ est une variété graphée constituée par collage, le long d'une famille de tores incompressibles, de blocs de l'un des trois types suivants:

1. un tore épais ;

2. un voisinage de bouteille de Klein;

3. un fibré en cercles au-dessus d'une surface compacte de caractéristique inférieure ou égale à -1 et non nécéssairement orientable. Toute composante fibrée $M$ de ce type a un bord qui intersecte non trivialement $\partial N$, et les courbes de $\Gamma_{(\partial N) \cap M}(\zeta)$ sont isotopes aux fibres de $M$.

Remarque 4.13. La variété $N$ n'est pas forcément connexe. 
Démonstration. À chaque fois que deux composantes $T^{2} \times I$ font bord commun, elles peuvent être regroupées pour former un unique $T^{2} \times I$. Si on coupe $N$ le long de l'union des $T^{2} \times\left\{\frac{1}{2}\right\}$ donnés par la proposition 4.9, alors les composantes connexes sont de l'un des trois types recherchés. Le cas 3 apparaît pour les composantes connexes qui contiennent un produit $A \times I$. Sur chaque composante $M$ de ce découpage qui est de la forme 3 , les fibres sont isotopes aux courbes $\partial A \times\{*\}$ des produits $A \times I$ qui la composent et qui sont de degré nul. Comme les anneaux $(\partial A) \times I$ contiennent une courbe de singularités isotope à leur âme (et donc aux fibres de $M$ d'après l'assertion 4.2), les courbes de $\Gamma_{\partial N}(\zeta) \cap M$ sont isotopes aux fibres.

Lemme 4.14. Soit $T \subset N$ un tore de recollement entre deux composantes fibrées $M$ et $M^{\prime}$ de type 3 le long duquel les fibrations ne correspondent pas. Il existe un voisinage tubulaire $T \times[-1,1]$ de $T=T \times\{0\}$ inclus dans $M \cup M^{\prime}$ et des structures $\zeta_{1}, \ldots, \zeta_{k}$ sur $T \times[-1,1]$ pour lesquelles les tores $T \times\{ \pm 1\}$ sont convexes, les composantes des courbes de découpage $\Gamma_{T \times\{ \pm 1\}}\left(\zeta_{i}\right)$ sont respectivement deux fibres de $M^{\prime}$ et pour lesquelles, pour tout $\xi \in \mathcal{T}(N, \zeta)$, il existe une isotopie de $\xi$ en $\xi^{\prime}$ dans $V$ relative $\grave{a} V \backslash \operatorname{Int}(N)$ qui fait coïncider $\left.\xi^{\prime}\right|_{T \times[-1,1]}$ avec une des structures $\zeta_{i}$.

Démonstration. On commence par prouver le fait suivant.

Lemme 4.15. Il existe un épaississement $T \times[-2,2]$ de $T$ dans $M \cup M^{\prime}$ tel que toute composante de $\Gamma_{T \times\{-2\}}(\xi)$ soit isotope à une fibre de $M$ et toute composante de $\Gamma_{T \times\{2\}}(\xi)$ soit isotope à une fibre de $M^{\prime}$.

Démonstration. Les intersections $\partial M \cap \partial N$ et $\partial M^{\prime} \cap \partial N$ sont non vides et contiennent une courbe de singularités isotope aux fibres. On prend des copies parallèles de ces courbes singulières dans $\operatorname{Int}(M)$ et $\operatorname{Int}\left(M^{\prime}\right)$. Ce sont des courbes legendriennes isotopes aux fibres dont l'enroulement (calculé par rapport à la fibration) est nul. On choisit alors un épaississement $T \times[-2,2]$ de $T=T \times\{0\}$ qui incorpore ces courbes legendriennes dans son bord. La condition d'enroulement nul fait que celles-ci peuvent être réalisées, après isotopie éventuelle, comme des orbites non singulières de $\xi T \times\{ \pm 2\}$. Elles y imposent donc la condition voulue sur $\Gamma_{T \times\{ \pm 2\}}(\xi)$.

D'après la classification des structures tendues sur le tore épais Gi4, Ho1, si les pentes des courbes de découpage au bord de $T \times[-2,2]$ ne coïncident pas, on peut réduire à deux le nombre de leurs composantes. On peut donc, sous les hypothèses du lemme 4.14 et les conclusions du lemme 4.15, isotoper toute structure $\left.\xi\right|_{T \times[-2,2]}$ relativement au bord de $T \times[-2,2]$ en $\xi^{\prime}$ de sorte que les découpages $\Gamma_{T \times\{ \pm 1\}}\left(\xi^{\prime}\right)$ aient deux composantes isotopes aux fibres de $M$ et de $M^{\prime}$. On peut également obtenir que la torsion de $\left.\xi^{\prime}\right|_{T \times[-1,1]}$ soit nulle. Il n'y a qu'un nombre fini de structures de contact tendues sur $T \times[-1,1]$ qui satisfassent ces conditions. D'où les structures $\zeta_{1}, \ldots, \zeta_{k}$ recherchées.

On a un résultat dans le même esprit pour les composantes $N(K)$. On rappelle que la bouteille de Klein possède seulement quatre classes d'isotopie distinctes de courbes fermées simples essentielles et non orientées. Précisément, dans un système de coordonnées $(y, t) \in(\mathbb{R} / \mathbb{Z}) \times[0,1]$ où $K \simeq(\mathbb{R} / \mathbb{Z}) \times[0,1] /(y, 0) \sim(-y, 1)$, on obtient quatre représentants avec les courbes $\gamma_{1}=\{t=0\}, \gamma_{2}=\{y=0\}, \gamma_{3}=\left\{y=\frac{1}{2}\right\}$ et $\gamma_{4}=\left\{y=\frac{1}{4}, y=\frac{3}{4}\right\}$. Similairement, $N(K)$ possède, à isotopie près, deux fibrations de Seifert différentes : une fibration en cercles sur la bande de Möbius dont les fibres sont isotopes à $\gamma_{1}$ et une fibration de Seifert sur le disque dont les fibres régulières sont isotopes à $\gamma_{4}$ et les deux fibres singulières à $\gamma_{2}$ et $\gamma_{3}$. 
Lemme 4.16. Soit $T$ un tore de recollement entre une composante fibrée $M$ de type 3 et un voisinage $N(K)$. Si aucune des deux fibrations possibles de $N(K)$ ne prolonge celle de $M$, alors il existe un voisinage $T \times[-1,1]$ de $T=T \times\{0\}$ dans $M \cup N(K)$ et une famille $\zeta_{1}, \ldots, \zeta_{k}$ de structures de contact sur $T \times[-1,1]$ avec les propriétés suivantes :

- les tores $T \times\{ \pm 1\}$ sont $\zeta_{i}$-convexes et les courbes de découpages $\Gamma_{T \times\{ \pm 1\}}\left(\zeta_{i}\right)$ sont respectivement deux fibres de $M$ et de $N(K)$ (pour une des deux fibrations de $N(K)$ ) ;

- toute structure $\xi \in \mathcal{T}(N, \zeta)$ est isotope à une structure $\xi^{\prime}$ égale à l'une des structures $\zeta_{i}$ sur $T \times[-1,1]$ par une isotopie qui est stationnaire en dehors de $N$.

Démonstration. On commence par normaliser toute structure $\xi \in \mathcal{T}(N, \zeta)$ au voisinage de $K$. Pour cela, on rend, par isotopie de $\xi$, la courbe $\gamma_{1}$ legendrienne, puis on maximise son invariant de Thurston-Bennequin relatif à $K$, parmi ceux qui sont négatifs ou nuls. On distingue deux cas.

Si ce nombre de Bennequin maximal est nul, alors $\gamma_{1}$ est, après isotopie de $\xi$, une courbe non singulière du feuilletage caractéristique de $K$. On peut alors trouver un voisinage $T \times[-2,2]$ de $T=T \times\{0\}$ dans $M \cup N(K)$ pour lequel le feuilletage caractéristique $\xi T \times\{2\}$ contient une courbe non singulière isotope à $\gamma_{1}$, c'est-à-dire aux fibres de la fibration de $N(K)$ sur la bande de Möbius, et $\xi T \times\{-2\}$ contient une courbe non singulière isotope aux fibres de $M$. Comme on l'a déjà vu précédemment, la classification des structures tendues sur le tore épais permet, dans le cas où ces deux directions ne sont pas isotopes dans $T^{2} \times[-2,2]$, d'isotoper $\xi$ dans $T \times[-2,2]$ pour l'amener sur un nombre fini de modèles fixés au préalable dans $T \times[-1,1]$, tous possédant les propriétés requises dans les conclusions du lemme 4.16.

Lorsque le nombre de Bennequin maximal de $\gamma_{1}$ est strictement négatif, on isotope $K$ pour que $\xi$ ne fasse pas de demi-tour inversé le long de $\gamma_{1}$. On découpe alors $K$ selon $\gamma_{1}$. Il apparaît un anneau $A$ à bord legendrien que l'on rend convexe par isotopie $\mathcal{C}^{\infty}$-petite relative à $\partial A$. Le découpage de $A$ est alors constitué de traverses. Ces traverses donnent dans $K$ une multi-courbe $\Gamma$ qui joue le rôle de « courbe de découpage » de $K$. Ici, on observe que chacune des composantes de $\Gamma$ est isotope à $\gamma_{2}, \gamma_{3}$ ou $\gamma_{4}$. De plus, le lemme de réalisation appliqué sur $A$ permet de dessiner sur $K$ des courbes legendriennes non singulières parallèles aux composantes de $\Gamma$. Celles-ci sont isotopes aux fibres régulières de la fibration de $N(K)$ sur le disque et peuvent être incorporées dans le bord d'un voisinage de $T$. On conclut alors comme précédemment.

Démonstration de la proposition 4.11. Si $\partial N=\emptyset$, alors soit $N(=V)$ est un fibré en tores sur le cercle, soit $N$ est obtenu en recollant deux voisinages $N(K)$ le long de leur bord. Le cas des fibrés en cercles est traité dans Gi4] et [Ho2]. On y obtient le théorème 0.6 et donc on peut prendre $N_{i}=\emptyset$ dans la proposition 4.11. Celui où $V=N=N(K) \cup N(K)$ s'étudie de manière similaire.

Proposition 4.17. Soit $V=N\left(K_{1}\right) \cup N\left(K_{2}\right)$ où $K_{1}$ et $K_{2}$ sont des bouteilles de Klein. L'espace des structures de contact tendues de torsion inférieure à $n \in \mathbb{N}$ possède un nombre fini d'orbites sous l'action du groupe engendré par les isotopies et les twists de Dehn.

Esquisse de démonstration. Soit $\xi$ une structure de contact tendue sur $V$. On normalise le « découpage » de $K_{1}$ puis celui de $K_{2}$ en minimisant le nombre de leurs composantes. On choisit des petits voisinages « homogènes $» N_{\varepsilon}\left(K_{i}\right)$ de $K_{i}$ et on identifie l'adhérence de leur complémentaire à $T \times[-2,2]$. Les découpages $\Gamma_{\partial N_{\varepsilon}\left(K_{i}\right)}$ sont constitués de fibres de l'une des fibrations des $N\left(K_{i}\right)$. Si aucune des deux fibrations de $N\left(K_{1}\right)$ ne s'étend 
à $N\left(K_{2}\right)$, alors on peut trouver une isotopie de $\xi$ en $\xi^{\prime}$ pour laquelle les découpages $\Gamma_{T \times\{ \pm 1\}}\left(\xi^{\prime}\right)$ sont constitués de deux fibres de la composante $N\left(K_{i}\right)$ correspondante. Si le nombre de composantes de $\Gamma_{\partial N_{\varepsilon}\left(K_{i}\right)}$ n'était pas deux, on trouverait une rocade s'appuyant sur $\partial N_{\varepsilon}\left(K_{i}\right)$ et par suite une rocade s'appuyant sur $K_{i}$ qui permettrait de réduire son découpage. D'où une contradiction. On peut ainsi se ramener au cas où toutes les structures considérées coïncident au voisinage des bouteilles $K_{i}$. La proposition découle alors de la classification des structures tendues sur le tore épais.

Le cas restant est celui où $V$ est un fibré de Seifert sur la bouteille de Klein, l'espace projectif où la sphère, avec respectivement 0,2 et 4 fibres singulières. Pour toute structure tendue $\xi$, la variété $V$ possède de plus une fibre régulière d'enroulement nul. On excise un voisinage standard d'une telle fibre régulière pour ce ramener au cas d'un fibré en cercles sur une surface à bord, lequel est traité dans la section 4.4.

On suppose maintenant que $\partial N \neq \emptyset$. Si deux composantes de type 3 de $N$ sont adjacentes le long d'un tore $T$, soit leurs fibrations coïncident au bord, et on les recolle pour former une composante fibrée élargie, soit elles sont différentes et, à l'aide du lemme 4.14, on se ramène à l'étude d'une famille de structures tendues sur le découpage de $N$ par $T \times[-1,1]$ qui coïncident au bord. Comme $\partial N \neq \emptyset$, toute composante $N(K)$, voisinage d'une bouteille de Klein $K$, est alors isolée ou adjacente à une composante $M$ de type 3. Dans le deuxième cas, le lemme 4.16 permet, quitte à effectuer un découpage le long du tore de recollement $T$ (et après isotopie des structures), de se ramener aux cas de $M$ et d'une composante $N(K)$ isolée ou de supposer que la fibration de $M$ s'étend en une fibration (de Seifert) sur $M \cup N(K)$. Pour obtenir la proposition 4.11, les composantes $N(K)$ isolées peuvent être traitées à part, de la même façon que dans la proposition 4.17.

Après ces opérations de réduction, la sous-variété $N$ est un fibré de Seifert au-dessus d'une surface compacte à bord non vide. Pour toute structure $\xi \in \mathcal{T}(N, \zeta)$, le fibré $N$ possède une fibre régulière d'enroulement nul. On se ramène au cas où $N$ est un fibré en cercles en retirant à $N$ des voisinages normalisés $N\left(\gamma_{1}\right), \ldots, N\left(\gamma_{l}\right)$ des fibres singulières (rendues legendriennes au préalable) $\gamma_{1}, \ldots, \gamma_{l}$. Pour cela, on incorpore dans le bord de chaque $N\left(\gamma_{i}\right)$ une fibre régulière d'enroulement nul qui forme une orbite périodique de $\xi \partial N\left(\gamma_{i}\right)$. Ceci impose que $\Gamma_{\partial N\left(\gamma_{i}\right)}$ est constitué de courbes isotopes aux fibres. On peut également imposer que \# $\left(\Gamma_{\partial N\left(\gamma_{i}\right)}\right)=2$ et d'après le théorème de classification des structures tendues sur le tore solide [Gi4, Ho1], il y a un nombre fini de structures tendues sur $N\left(\gamma_{i}\right)$ qui ont un tel découpage au bord.

Enfin, maintenant que l'on s'est ramené à un fibré en cercles, on remarque que, à isotopie près, toutes les composantes des courbes de découpage présentes au bord sont $\mathbf{S}^{1}$-invariantes. En conclusion, on obtient la proposition 4.11.

\subsection{Le cas des fibrés en cercles}

Pour conclure la preuve du théorème 0.6, on utilise des résultats de [Gi4, Gi5] et [Ho2] qui classifient les structures de contact tendues sur les fibrés en cercles : l'ensemble $\mathcal{T}(N, \zeta)$ contient un nombre fini de structures de contact tendues de torsion donnée, à conjuguaison près par des produits de twists de Dehn le long de tores incompressibles dans $N$ et d'isotopies. La classification est même plus précise.

Théorème 4.18. Gi4, Gi5, Ho2 Soit $S$ une surface compacte orientée de bord non vide et $E$ un ensemble formé d'un nombre pair (et non nul) de points sur chaque composante de $\partial S$. Soit également $\zeta$ un germe de structure de contact le long de $\partial M$. On suppose que $\partial M$ 
est $\zeta$-convexe et que sa courbe de découpage est $S^{1} \times E$. Les classes d'isotopies de structures de contact tendues sur $M$ qui cö̈ncident avec $\zeta$ sur le bord sont en correspondance bijective avec les sous-variétés incompressibles (mais pas nécéssairement $\partial$-incompressibles) de dimension un $\Gamma$ de $S$ avec $\partial \Gamma=E$.

Dans ce théorème, il faut voir $\Gamma$ comme la courbe de découpage « minimale » d'une section $\{0\} \times S$ rendue convexe au préalable. Lorsque $\zeta$ est $S^{1}$-invariante, alors toutes les structures tendues sur $M$ qui coïncident avec $\zeta$ au bord le sont également (à isotopie près).

Dans le cas qui nous intéresse cependant, la base $S$ de $\pi: N \rightarrow S$ n'est pas toujours orientable. On se donne une multi-courbe compacte plongée dans $S$ dont le complémentaire est orientable et dont la préimage par $\pi$ est une union disjointe de bouteilles de Klein. Pour toute structure $\xi \in \mathcal{T}(N, \zeta)$, on peut réaliser une isotopie de $\xi$ à support dans $N$ pour que chacune de ces bouteilles $K$ contienne une fibre de $\pi$ d'enroulement nul qui est une orbite non singulière $\gamma$ de son feuilletage caratéristique. La surface obtenue en découpant $K$ selon $\gamma$ est un anneau que l'on peut rendre convexe par perturbation $\mathcal{C}^{\infty}$ petite de $\xi$ relative à $\gamma$. Par extension, on considère que la bouteille $K$ est « convexe » et que son découpage s'identifie à celui de $A$. Celui-ci est consitué, après isotopie, d'une union de fibres de $\pi$. En appliquant le lemme de réalisation de feuilletages à $A$, on arrive sans difficulté à conjuguer la structure $\xi$ sur un voisinage $N(K)$ de $K$ à la structure d'équation $\sin ((2 n+1) \pi t) d x+\cos ((2 n+1) \pi t) d y=0$ pour l'identification donnée par

$$
N(K) \simeq\{(x, y, t) \in[-1,1] \times \mathbb{R} / \mathbb{Z} \times[0,1]\} /(x, y, 0) \sim(-x,-y, 1)
$$

où $K=\{x=0\}$.

Sur la section $B_{0}=\{y=0\}$ de $\pi: N(K) \rightarrow B$, on peut matérialiser une courbe de découpage par les $2 n+1$ traverses $\left\{y=0, t=\frac{2 k+1}{4 n+2}\right\}, k \in\left[0,2 n+1\right.$ [. Même si $B_{0}$ n'est pas orientable, ce découpage prend un sens lorsqu'on considère le disque à bord legendrien $B_{1}$ obtenu en découpant $B_{0}$ le long de $\{t=0\}$. La structure $\xi$ est $S^{1}$-invariante au-dessus de $B_{1}$.

Dans le complémentaire de l'union de ces voisinages de bouteilles de Klein normalisés, qui est le produit $M_{0}$ d'une surface compacte orientable $S_{0}$ par le cercle, on minimise, comme dans le théorème 4.18, le découpage d'une section $\{0\} \times S_{0}$ dont le bord est égal à celui des bandes de Möbius $B_{0}$ trouvées dans chaque $N(K)$. Comme elle l'est sur les composantes de $\partial M_{0}$ (après utilisation du lemme de réalisation de feuilletages), la structure $\xi$ est, d'après le théorème 4.18, (isotope à une structure) $S^{1}$-invariante sur $S^{1} \times S_{0}$. On recolle la courbe de découpage obtenue sur $\{0\} \times S_{0}$ avec les arcs tracés sur les $B_{0}$. On obtient ainsi une « courbe de découpage $» \Gamma_{S}(\xi)$ de $S$. Soit $k(\xi)$ le nombre de composantes de $\Gamma_{S}(\xi)$.

Si $k(\xi)$ est borné indépendemment de $\xi \in \mathcal{T}(N, \zeta)$, alors toutes les configurations possibles pour la famille $\left(\Gamma_{S}(\xi)\right)_{\xi \in \mathcal{T}(N, \zeta)}$ se déduisent d'un nombre fini d'entre elles par des twists de Dehn et des isotopies de $S$. Comme $\xi$ est déterminée par $\Gamma_{S}(\xi)$ et que tout twist de Dehn sur $S$ provient d'un twist le long d'un tore de $N$, on obtient la conclusion.

Dans le cas contraire, pour tout $p \geq 0$, il existe une structure $\xi_{p} \in \mathcal{T}(N, \zeta)$ pour laquelle la courbe de découpage $\Gamma_{S}\left(\xi_{p}\right)$ contient une famille de $p$ courbes parallèles. Celleci est incluse dans un anneau $A \subset S$. La fibration est triviale au-dessus de $A$, et $\operatorname{sur} \pi^{-1}(A)$ la structure $\xi_{p}$ est $S^{1}$-invariante. On vérifie facilement que le tore épais $\left(\pi^{-1}(A),\left.\xi\right|_{\pi^{-1}(A)}\right)$ contient un tore de torsion $\left[\frac{p}{2}\right]-1$, d'où une contradiction. 


\section{$5 \quad$ Finitude pour les nouds legendriens}

Pour finir, on démontre ici le théorème 0.10 . Soit $\mathcal{K}$ une classe topologique de nœuds dans $\mathbf{S}^{3}$ et $K$ un de ses représentants. On note $\zeta_{0}$ la structure de contact standard sur $\mathbf{S}^{3}, V$ la variété à bord $\mathbf{S}^{3} \backslash \operatorname{Int}(N(K))$ et $\mathcal{K}_{\zeta_{0}, n}$ l'ensemble des nœuds legendriens de $\left(\mathbf{S}^{3}, \zeta_{0}\right)$ dans la classe $\mathcal{K}$ dont l'invariant de Thurston-Bennequin vaut $n$. Tout nœud $L \in \mathcal{K}_{\zeta_{0}, n}$ possède un voisinage tubulaire $N(L)=\mathbf{D}^{2} \times \mathbf{S}^{1}$ contenant $L$ comme $\{0\} \times \mathbf{S}^{1}$ et « standard $»$ au sens où $\zeta_{0}$ y est définie par

$$
\sin \theta d x+\cos \theta d y=0, \quad(x, y, \theta) \in \mathbf{D}^{2} \times \mathbf{S}^{1} .
$$

Pour tout $L \in \mathcal{K}_{\zeta_{0}, n}$, la restriction de $\zeta_{0}$ à $\mathbf{S}^{3} \backslash \operatorname{Int}(N(L))$ donne une structure de contact $\xi_{L}$ sur $V$. La feuilletage caractéristique $\xi_{L} \partial V$ est déterminé, à isotopie près, par la pente de ses lignes singulières. Cette pente elle-même est déterminée par l'invariant de ThurstonBennequin $n$ de $L$. On peut donc supposer que toutes les structures $\xi_{L}, L \in \mathcal{K}_{\zeta_{0}, n}$, coïncident le long de $\partial V$. Or la variété $V$ est irréductible et les structures $\xi_{L}$ sont tendues et de torsion nulle : d'après le théorème 0.8 , elles vivent dans un nombre fini de classes, modulo isotopie et conjugaison par des twists de Dehn sur des tores. Vus dans $\mathbf{S}^{3}$, ces twists sont isotopes à l'identité. Si $\xi_{L}$ et $\xi_{L^{\prime}}$ sont dans une même classe, il existe donc une isotopie de $\mathbf{S}^{3}$ issue de l'identité dont le temps 1 envoie $L$ sur $L^{\prime}$ et préserve $\zeta_{0}$. Comme l'espace des structures de contact tendues positives sur $\mathbf{S}^{3}$ est simplement connexe [El2], cette isotopie peut être déformée en une isotopie de contact qui amène $L$ sur $L^{\prime}$. C'est une isotopie legendrienne entre $L$ et $L^{\prime}$.

Question 5.1. Dans une classe d'isotopie de nœuds donnée, existe-t-il un nombre fini de nœuds legendriens dont tout nœud legendrien se déduise par isotopie legendrienne et stabilisation?

\section{Références}

[Be] D. Bennequin, Entrelacements et équations de Pfaff. Astérisque 107-108 (1983), 87-161.

[Br] M. Brittenham, Essential laminations and Haken normal forms. Pac. J. Math. 168 (1995), 217-234.

[Co1] V. Colin, Chirurgies d'indice un et isotopies de sphères dans les variétés de contact tendues. C. R. Acad. Sci. Paris Sér. I Math. 324 (1997), 659-663.

[Co2] V. Colin, Sur la torsion des structures de contact tendues. Ann. Sci. Éc. Norm. Sup. 34 (2001), 267-286.

[Co3] V. Colin, Une infinité de structures de contact tendues sur les variétés torö̈dales. Comment. Math. Helv. 76 (2001), 353-372.

[Co4] V. Colin, Structures de contact tendues sur les variétés torö̈dales et approximation de feuilletages sans composante de Reeb. Topology 41 (2002), 1017-1029.

[CGH1] V. Colin, E. Giroux et K. Honda, On the coarse classification of tight contact structures. Proceedings of Symposia in Pure Mathematics 71 (2003), 109-120.

[El1] Y. Eliashberg, Classification of over-twisted contact structures on 3-manifolds. Invent. Math. 98 (1989), 623-637. 
[El2] Y. Eliashberg, Contact 3-manifolds twenty years since J. Martinet's work. Ann. Inst. Fourier 42 (1992), 165-192.

[El3] Y. Eliashberg, Filling by holomorphic discs and its applications. London Math. Soc. Lect. Notes Ser. 151 (1991), 45-67.

[ET] Y. Eliashberg et W. Thurston, Confoliations. University Lecture Series 13, Amer. Math. Soc. 998).

[Et] J. Etnyre, Tight contact structures on lens spaces. Commun. Contemp. Math. 2 (2000), 559-577.

[EH1] J. Etnyre et K. Honda, On the non-existence of tight contact structures. Ann. of Math. 153 (2001), 749-766.

[EH2] J. Etnyre et K. Honda, Tight contact structures with no symplectic fillings. Invent. Math. 148 (2002), 609-626.

[FO] W. Floyd et U. Oertel, Incompressible surfaces via branched surfaces. Topology 23 (1984), 117-125.

[Ga] D. Gabai, Essential laminations and Kneser normal form. J. Diff. Geom. 53 (1999), 517-574.

[Gi1] E. Giroux, Convexité en topologie de contact. Comment. Math. Helv. 66 (1991), $637-677$.

[Gi2] E. Giroux, Une structure de contact, même tendue, est plus ou moins tordue. Ann. Sci. Éc. Norm. Sup. 27 (1994), 697-705.

[Gi3] E. Giroux, Une infinité de structures de contact tendues sur une infinité de variétés. Invent. Math. 135 (1999), 789-802.

[Gi4] E. Giroux, Structures de contact en dimension trois et bifurcations des feuilletages de surfaces. Invent.Math. 141 (2000), 615-689.

[Gi5] E. Giroux, Structures de contact sur les variétés fibrées en cercles au-dessus d'une surface. Comment. Math. Helv. 76 (2001), 218-262.

[Gi6] E. Giroux, Structures de contact, livres ouverts et tresses fermées. En préparation.

[Gr] M. Gromov, Pseudoholomorphic curves in symplectic manifolds. Invent. Math. 82 (1985), 307-347.

[Ha] W. Haken, Theorie der Normalflächen. Ein Isotopiekriterium für den Kreisknoten. Acta Math. 105 (1961), 245-375.

[Ho1] K. Honda, On the classification of tight contact structures I. Geom. Topol. 4 (2000), 309-368.

[Ho2] K. Honda, On the classification of tight contact structures II. J. Diff. Geom. 55 (2000), 83-143.

[Ho3] K. Honda, Gluing tight contact structures. Duke Math. J. 115 (2002), 435-478.

[HKM] K. Honda, W. Kazez et G. Matić, Convex decomposition theory. Internat. Math. Res. Notices 2002, 55-88.

[Ka] Y. Kanda, The classification of tight contact structures on the 3-torus. Comm. Anal. Geom. 5 (1997), 413-438.

[Kn] H. Kneser, Geschlossene Flächen in dreidimensionalen Mannigfaltigkeiten. Jahres. der Deut. Math.-Verein. 38 (1929), 248-260. 
[KM] P. Kronheimer et T. Mrowka, Monopoles and contact structures. Invent. Math. 130 (1997), 209-255.

[Li] T. Li, Laminar branched surfaces in 3-manifolds. Geom. Topol. 6 (2002), 153194.

[LS] P. Lisca et A. Stipsicz, An infinite family of tight, not semi-fillable contact threemanifolds. Geom. Topol. 7 (2003), 1055-1073.

[Ma] S. MakAR-Limanov, Morse surgeries of index 0 on tight manifolds. Prépublication (1997).

[Th] W. Thurston, A norm for the homology of 3-manifolds. Mem. Amer. Math. Soc. 59 (1986), 99-130.

[Wa] F. Waldhausen, On irreducible 3-manifolds which are sufficiently large. Ann. of Math. 87 (1968), 56-88.

[Wh] J. H. C. Whitehead, On $\mathcal{C}^{1}$ complexes. Ann. of Math. 41 (1940), 809-824.

Université de Nantes, UMR 6629 du CNRS, 44322 Nantes, France

Vincent.Colin@math.univ-nantes.fr

École normale supérieure de Lyon, 69000 Lyon, France

Emmanuel.Giroux@umpa.ens-lyon.fr

University of Southern California, Los Angeles, CA 90089

khonda@math.usc.edu

http ://rcf.usc.edu/ khonda 